\title{
Host galaxies of high-redshift quasars: SMBH growth and feedback
}

\author{
Milena Valentini ${ }^{1,2,3,4 \star}$, Simona Gallerani ${ }^{3}$, and Andrea Ferrara ${ }^{3}$ \\ ${ }^{1}$ Universitäts-Sternwarte, Fakultät für Physik, Ludwig-Maximilians-Universität München, Scheinerstr. 1, 81679 München, Germany \\ ${ }^{2}$ Excellence Cluster ORIGINS, Boltzmannstr. 2, D-85748 Garching, Germany \\ ${ }^{3}$ Scuola Normale Superiore, Piazza dei Cavalieri 7, I-56126 Pisa, Italy \\ ${ }^{4}$ INAF - Osservatorio Astronomico di Trieste, via Tiepolo 11, I-34131 Trieste, Italy
}

Accepted 2021 July 9. Received 2021 June 21; in original form 2020 December 26.

\begin{abstract}
The properties of quasar-host galaxies might be determined by the growth and feedback of their supermassive $\left(\mathrm{SMBH}, 10^{8-10} \mathrm{M}_{\odot}\right)$ black holes. We investigate such connection with a suite of cosmological simulations of massive (halo mass $\approx 10^{12} \mathrm{M}_{\odot}$ ) galaxies at $z \simeq 6$ which include a detailed sub-grid multiphase gas and accretion model. BH seeds of initial mass $10^{5} \mathrm{M}_{\odot}$ grow mostly by gas accretion, and become SMBH by $z=6$ setting on the observed $M_{\mathrm{BH}}-M_{\star}$ relation without the need for a boost factor. Although quasar feedback crucially controls the SMBH growth, its impact on the properties of the host galaxy at $z=6$ is negligible. In our model, quasar activity can both quench (via gas heating) or enhance (by ISM over-pressurization) star formation. However, we find that the star formation history is insensitive to such modulation as it is largely dominated, at least at $z>6$, by cold gas accretion from the environment that cannot be hindered by the quasar energy deposition. Although quasar-driven outflows can achieve velocities $>1000 \mathrm{~km} \mathrm{~s}^{-1}$, only $\approx 4 \%$ of the outflowing gas mass can actually escape from the host galaxy. These findings are only loosely constrained by available data, but can guide observational campaigns searching for signatures of quasar feedback in early galaxies.
\end{abstract}

Key words: galaxies: quasars: supermassive black holes; galaxies: formation; galaxies: evolution; galaxies: ISM; methods: numerical.

\section{INTRODUCTION}

Quasars are among the most luminous astrophysical sources: they shine at the centre of their host galaxies, where gas accretion fuels a supermassive black hole (SMBH), their engine. Quasar high luminosity allows their identification out to very-high redshift $(z \gtrsim 6)$ : they can be deemed as beacons in the early universe, thus being signposts of the early steges of galaxy evolution and black hole (BH) growth. More than 200 quasars have been discovered over the last decades at $z \gtrsim 6$ by means of optical/near-infrared (IR) surveys (e.g. Fan et al. 2006; Willott et al. 2010; Mortlock et al. 2011; Venemans et al. 2013, 2015; Bañados et al. 2014; Jiang et al. 2016; Matsuoka et al. 2016, 2019b; Pons et al. 2019), and have been studied through their ultraviolet (UV) and X-ray emission (Brandt et al. 2002; Farrah et al. 2004; Shemmer et al. 2006; Page et al. 2014; Gallerani et al. 2017b; Koptelova et al. 2017; Nanni et al. 2017, 2018; Salvestrini et al. 2019; Vito et al. 2019; Pons et al. 2020). These observations have provided information about the physical properties of these powerful active galactic nuclei (AGN), characterized by bolometric

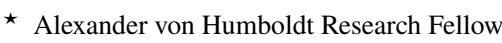

E-mail: valentini@usm.lmu.de
}

luminosities $L_{\text {bol }} \gtrsim 10^{46} \mathrm{erg} / \mathrm{s}$ (Willott et al. 2010; De Rosa et al. 2014; Barnett et al. 2015; Venemans et al. 2015; Gallerani et al. 2017b; Mazzucchelli et al. 2017; Matsuoka et al. 2019a). In particular, it has been found that $z \sim 6$ quasars are powered by SMBHs with typical masses spanning the range $10^{8}-10^{10} \mathrm{M}_{\odot}$ (e.g. Ho 2007; Wang et al. 2010; Venemans et al. 2016; Pensabene et al. 2020). The advent of the Atacama Large Millimeter/submillimeter Array (ALMA) has later allowed to investigate the properties of the host galaxies of these distant quasars (e.g. Carilli \& Walter 2013; Wang et al. 2013; Venemans et al. 2016, 2017a,b, 2019; Gallerani et al. 2017a; Willott et al. 2017; Decarli et al. 2018; Feruglio et al. 2018; Carniani et al. 2019; Novak et al. 2019; Wang et al. 2019).

The presence of SMBHs which grew as massive as $10^{8}-$ $10^{10} \mathrm{M}_{\odot}$ in less than $\sim 1 \mathrm{Gyr}$ (i.e. the age of the universe at $z \simeq 6$ ) represents an important constraint for SMBH fromation channels, and poses a challenging question from a theoretical perspective (see e.g. Volonteri 2010; Volonteri \& Bellovary 2012; Latif \& Ferrara 2016; Gallerani et al. 2017a; Mayer \& Bonoli 2019, for reviews, and references therein).

In particular, two among the several facets which are highly debated and still need to be addressed concern the initial seeds of these SMBHs and their maximum accretion rate. As for the initial 
seeds of SMBHs, the three most popular formation scenarios (for a review see Latif \& Ferrara 2016) are: (i) the core-collapse of massive, Pop III stars; (ii) the collapse of the innermost region of a dense star cluster; and (iii) the direct collapse BH channel (e.g. Bromm \& Loeb 2003; Begelman et al. 2006; Mayer et al. 2010; Ferrara et al. 2014; Pacucci \& Ferrara 2015; Maio et al. 2018). The aformentioned scenarios are still debated, and the likelihood of each of them is deeply connected (among other factors) to the redshift at which SMBH seeds formed and to the timescale over which they accreted gas to reach the mass they have at $z \sim 6$. Gas accretion should proceed at a fast pace, with $\mathrm{BH}$ accretion rates close to the Eddington accretion rate for long periods so as to let $\mathrm{BH}$ seeds reach their final mass by $z \sim 6$. In addition, the possibility of short-lived and intermittent episodes of super-critical accretion rate - where the $\mathrm{BH}$ accretion rate overshoots the Eddington limit - has been suggested to reconcile theoretical predictions with observations (Madau et al. 2014; Volonteri et al. 2015; Inayoshi et al. 2016; Regan et al. 2019). Besides gas accretion, the concurrent channel for BH growth is merging with other BHs.

Moreover, the problem of how BHs grow supermassive in the early universe is deeply intertwined both with the assessment of the contribution from AGN to the reionization of the universe (Volonteri \& Gnedin 2009; Giallongo et al. 2015; Onoue et al. 2017; Hassan et al. 2018; Meyer et al. 2019; Trebitsch et al. 2020b), and with the early stages of BH-galaxy co-evolution (e.g. Lamastra et al. 2010; Merloni et al. 2010; Sarria et al. 2010; Willott et al. 2010; Portinari et al. 2012; Bongiorno et al. 2014; Valiante et al. 2014; Volonteri \& Reines 2016; Pensabene et al. 2020).

High-redshift galaxies are complex ecosystems: they indeed have a multiphase interstellar medium (ISM), with gas spanning a wide range of temperatures, densities, and ionization states (e.g. Wolfe et al. 2005; Carilli \& Walter 2013; Dayal \& Ferrara 2018, for reviews, and references therein). As for $z \geq 6$ quasar-host galaxies, observations show that they typically have dynamical (gas and stellar) masses in the range $\sim 10^{10}-10^{11} \mathrm{M}_{\odot}$, star formation rates (SFRs) from few hundreds to few thousands $\mathrm{M}_{\odot} \mathrm{yr}^{-1}$ (Maiolino et al. 2005; Solomon \& Vanden Bout 2005; Wang et al. 2016; Trakhtenbrot et al. 2017a; Willott et al. 2017; Decarli et al. 2018; Venemans et al. 2018; Bischetti et al. 2019; Shao et al. 2019), molecular gas ( $>10^{10} \mathrm{M}_{\odot}$ Walter et al. 2004; Shields et al. 2006; Venemans et al. 2017b; Combes 2018; Feruglio et al. 2018; Li et al. 2020), dust (> 10 ${ }^{8} \mathrm{M}_{\odot}$; e.g. Wang et al. 2008; Venemans et al. 2016; Carniani et al. 2019), and outflows (e.g. Maiolino et al. 2012; Cicone et al. 2015; Bischetti et al. 2019; Stanley et al. 2019). The cold and molecular gas phases play a key role, as they provide the reservoir of gas which fuels star formation (SF). The tight correlation between the SFR and the stellar mass (i.e. the main-sequence) is also already established at redshift $z \geq 6$ (Bouwens et al. 2012; Salmon et al. 2015): the normalization of this relation is observed to be higher than in the lower-redshift universe, thus implying higher SFRs and shorter gas depletion timescales for distant galaxies (Solomon \& Vanden Bout 2005; Daddi et al. 2010).

Another piece of evidence which adds complexity to this picture is the role of stellar and quasar ${ }^{1}$ feedback. Feedback is the complex set of processes by which SMBHs and supernovae ( $\mathrm{SNe}$ ) affect the evolution of their host galaxy and surrounding environment, and mainly develops through the injection of energy and momentum. These processes control structure formation and evolution across

1 The terms quasar feedback and AGN feedback are often used interchangeably in this work. cosmic time: for instance, they shape galaxy morphology, affect the properties of the ISM, and regulate (or even quench) SF in galaxies (e.g. McNamara \& Nulsen 2007; Fabian 2012; Kormendy \& Ho 2013). Feedback mechanisms can indeed prevent gas from being accreted or from effectively cooling (preventive feedback); they can remove gas from the innermost regions of forming structures where SF occurs (ejective feedback), or can suppress the SF efficiency (mainly via ISM heating and turbulence, e.g. Alatalo et al. 2015; Costa et al. 2018; but see Bischetti et al. 2020 for different evidence). Feedback processes play a key role in determining the stellar-to-halo mass fraction and reducing the baryon coversion efficiency (e.g. Guo et al. 2010; Moster et al. 2010; Behroozi et al. 2013; Genzel et al. 2015; Pillepich et al. 2018b; Bluck et al. 2020). As a direct consequence, the low- and high-mass end of the galaxy stellar mass function is lowered and the shape predicted by theoretical models and simulations better agree with observations (Croton et al. 2006; Puchwein \& Springel 2013; Hirschmann et al. 2014; Vogelsberger et al. 2014). Despite of the fact that one process can dominate over the others depending on the system properties and on cosmic time (e.g. Förster Schreiber et al. 2019), feedback mechanisms often occur in a simultaneous way, and it is hard to distinguish their imprints.

Quasar feedback is expected to be the most important mechanism to suppress SF in massive systems, and AGN-driven outflows represent one of the main signatures of ongoing AGN activity. Other processes can contribute to suppress SF, e.g. stellar feedback, morphological quenching or gravitational heating, but it is unlikely that these processes alone (i.e. without the inclusion of quasar feedback) can keep massive systems quiescent. On the other hand, stellar feedback and environmental processes play the main role in regulating the star formation history (SFH) of lower-mass systems $\left(\leq 10^{11} \mathrm{M}_{\odot}\right)$. Moreover, quasar feedback is fundamental to control the $\mathrm{BH}$ growth and the AGN activity itself, by regulating the evolution of physical properties of the gas surrounding the $\mathrm{BH}$, and thus of BH accretion and luminosity. However, it is still debated whether quasar feedback is the main driver of galaxy evolution and to what level it impacts on the physical properties of the bulk of the gas in galaxies. This is due to poor statistics and little availability of observations, especially at high redshift, where the SMBH activity or feedback is caught in the act (Veilleux et al. 2005; Fabian 2012; Fiore et al. 2017).

The challenge of exploring the assembly of high-redshift systems and reproducing the growth of their SMBHs in the early universe can be tackled through cosmological simulations, which represent a unique theoretical tool (e.g., among those focussing on the high-z universe, Dubois et al. 2012; Bellovary et al. 2013; Costa et al. 2014; Feng et al. 2014, 2016; Fiacconi et al. 2017; Olsen et al. 2017; Barai et al. 2018; Lupi et al. 2019; Trebitsch et al. 2020a). Simulations indeed allow to go through and connect subsequent evolutionary stages, rather than (observing) a single frame. Several numerical works have investigated interesting properties of high-redshift quasar-host galaxies in terms of e.g. their environment (Costa et al. 2014), the impact of quasar outflows on the host galaxy (Barai et al. 2018), and how cold flows from the large scale contribute to the growth of SMBHs (Feng et al. 2014). However, even if there is a general consensus on the key role played by SMBHs in the evolution of their host galaxy, details on the relative contribution of different processes in establishing final properties of systems are still debated. Moreover, predictions from simulations appear to be extremely sensitive to the different sub-resolution models adopted. As for the modelling of quasar feedback, for instance, models adopting kinetic injection of feedback energy (e.g. Barai et al. 2016, 2018) 
usually produce stronger feedback effects than those resulting from simulations assuming that feedback energy is either deposited as thermal only, or provided in a hybrid way. This difference mainly stems from the facts that kinetic energy is not radiated away when velocity is imparted to gas, and that in this case thermalisation happens by construction later and at larger scales (see e.g. discussion in Costa et al. 2020, and references therein). Cosmological simulations are undoubtedly crucial to test different scenarios and to assess the impact of different processes, as they allow the possibility of switching on and off different physical modules in the adopted code.

Another appealing feature of cosmological simulations as for the investigation of how SMBHs reach the observed mass at $z=6$ is that they allow to explore the relative contribution of gas accretion and $\mathrm{BH}-\mathrm{BH}$ merging to $\mathrm{BH}$ growth, and to quantify the impact of different assumptions for SMBH seed mass. Interestingly, for instance, Huang et al. (2020) showed that the adopted value of the $\mathrm{BH}$ seed mass shapes the $\mathrm{BH}$ early growth and merging history, even if the final mass of the SMBH at $z=6$ is not sensitive to the assumed seed mass. Unfortunately, state-of-the-art cosmological simulations to date usually adopt rather crude seeding prescriptions for $\mathrm{BHs}$, as they seed $\mathrm{BH}$ particles with a mass that is assumed to be already the rusult of the formation of SMBH seeds, whose details are not captured.

An additional task that cosmological simulations can accomplish is to shed some light on the progenitors of SMBHs observed at redshift $z \sim 6$. Indeed, to spot BHs as massive as $\sim 10^{7} \mathrm{M}_{\odot}$ in the distant universe has proven less successful so far: this questions if and why they are so rare, and whether they are possibly obscured or too faint to be detected by current facilities. This theoretical approach has also a twofold, paramount importance: results of simulations can be indeed used to interpret observational results and to guide future surveys.

The framework that we have just outlined opens to new challenging tasks. The goal of this paper is to: (a) investigate how BHs grow supermassive in the early universe, and (b) explore their impact on the host galaxy and surrounding ISM. To this aim, we introduce a new suite of cosmological hydrodinamical simulations, where we take advantage of a detailed modelling of both the ISM physics, and BH accretion and feedback. The main questions that we aim at addressing with this introductory work are the following: what is the impact of stellar and quasar feedback on the ISM of high-redshift $(z \simeq 6)$ galaxies hosting SMBHs? How does thermal quasar feedback affect the growth history of SMBHs in the early universe? Do SMBH properties correlate with those of the hosting galaxies? What is the relative contribution of stellar and quasar feedback in promoting outflows? Can we constrain galactic outflow features at high redshift?

The outline of this Paper is as follows. Section 2 introduces the set of cosmological simulations and describes the main features of the sub-resolution model adopted. In Sections 3 and 4, we present and discuss our results. We draw conclusions in Section 5.

\section{THE SUITE OF COSMOLOGICAL SIMULATIONS}

In this Section, we describe the initial conditions (Section 2.1) of our cosmological simulations, the sub-resolution model that we adopt (Section 2.2), and the set of simulations that we performed (Section 2.3). Simulations have been carried out with the TreePM+SPH (smoothed particle hydrodynamics) GADGET3 code, a non-public evolution of the GADGET2 code (Springel 2005). We adopt the advanced formulation of SPH presented in Beck et al. (2016) and introduced in cosmological simulations adopting our sub-resolution model by Valentini et al. (2017). This improved formulation of SPH features a high-ordel kernel function, an artificial conduction term, a correction for the artificial viscosity, and a wake-up scheme, among the main refinements.

\subsection{Initial conditions}

We used the MUSIC ${ }^{2}$ software (Hahn \& Abel 2011) to generate the initial conditions (ICs). The assumed $\Lambda \mathrm{CDM}$ cosmology is the following: $\Omega_{\mathrm{m}}=0.3089, \Omega_{\Lambda}=0.6911, \Omega_{\text {baryon }}=0.0486$, $\sigma_{8}=0.8159, n_{s}=0.9667$, and $H_{0}=100 \mathrm{hm} \mathrm{s}^{-1} \mathrm{Mpc}^{-1}=$ $67.74 \mathrm{~km} \mathrm{~s}^{-1} \mathrm{Mpc}^{-1}$. These parameters are in agreement with the results of Planck Collaboration et al. (2016). First, a parent, DMonly simulation of a cubic volume of size $L=148 \mathrm{cMpc}$ (i.e. comoving $\mathrm{Mpc})^{3}$ is run starting at $z=100$ down to $z=6$, with periodic boundary conditions. We used $512^{3} \mathrm{DM}$ particles, the resulting mass resolution being $9.4 \cdot 10^{8} \mathrm{M}_{\odot}$. The gravitational softening length is set to $5.8 \mathrm{ckpc}$ (corresponding to $1 / 50$ of the mean inter-particle spacing). At $z=6$, subhaloes have been identified by means of the SUBFIND algorithm (Springel et al. 2001; Dolag et al. 2009). The subhalo finder algorithm provides the mass of each subhalo, as well as the coordinates of its centre, which is represented by the position of the subhalo particle with the minimum value of the gravitational potential. The subhalo mass is used to compute the virial radius of the structure. The virial radius is defined as the radius that encloses an overdensity of $\Delta_{\text {vir }}(z)$ times the critical density of the universe at that redshift (Barkana \& Loeb 2001), where $\Delta_{\text {vir }}(z)$ is the overdensity of virialised structures in a flat universe (Bryan \& Norman 1998). The main properties of subhaloes, along with their distribution in the parent, DM-only simulation at $z=6$ are discussed in Appendix A.

We chose a DM subhalo and re-simulated it with a higher resolution, zoomed-in simulation. Our target DM subhalo has been selected among those subhaloes which are at least as massive as $10^{12} \mathrm{M}_{\odot}$ at $z=6$, so as to be the eligible halo of a quasar-host galaxy (see Appendix A for further details). The target subhalo has a mass of $1.12 \cdot 10^{12} \mathrm{M}_{\odot}$ and a virial radius of $r_{\mathrm{vir}, \mathrm{DM}}=48.1 \mathrm{pkpc}$. We identified the DM particles within $2.5 r_{\text {vir,DM }}$ at $z=6$ and traced them back to their initial positions at $z=100$. The positions occupied by these particles define a Lagrangian region of $144.4(\mathrm{cMpc})^{3}$. By approximating this volume with a cube, the side of the zoom-in volume is $5.25 \mathrm{cMpc}$. We increased the resolution of the ICs by adding three more levels of refinement within the Lagrangian region with the MUSIC software, and included baryons. In the final zoomed-in simulation, the highest resolution DM particles have a mass of $m_{\mathrm{DM}}=1.55 \cdot 10^{6} \mathrm{M}_{\odot}$, while gas particles have $m_{\text {gas }}=2.89 \cdot 10^{5} \mathrm{M}_{\odot}$. Softening lengths for high-resolution $\mathrm{DM}$ and baryonic particles are as follows: $\epsilon_{\mathrm{DM}}=0.72 \mathrm{ckpc}$ and $\epsilon_{\text {bar }}=0.41 \mathrm{ckpc}$, respectively, which translate in a force resolution of $\epsilon_{\mathrm{DM}}=103 \mathrm{ppc}$ and $\epsilon_{\mathrm{bar}}=59 \mathrm{ppc}$ (i.e. physical pc at $z=6$ ).

The ICs of the zoomed-in simulation have $\sim 2 \times 3.14 \cdot 10^{6}$ particles of gas and high-resolution DM, and $\sim 1.35 \cdot 10^{8}$ lowerresolution DM particles. We verified with a DM-only test run whose resolution is analogous to that of the zoomed-in simulation that the main subhalo at $z=6$ is not contaminated by lower-resolution

\footnotetext{
2 https://www-n.oca.eu/ohahn/MUSIC/

3 We use the letter $c$ before the corresponding unit to refer to comoving distances (e.g. ckpc), while by analogy the letter $p$ stands for physical units (e.g. pkpc). When not explicitly stated, we are referring to physical distances.
} 
DM particles. There are no lower-resolution DM particles within the virial radius of the main progenitor of the target subhalo at all redshifts; moreover, the fraction of contaminating particles within twice the virial radius of the main progenitor of the target subhalo is below $1 \%$ in mass and $0.1 \%$ in number. The ICs of the zoomed-in simulation are evolved until $z=6$ with different flavours of baryonic physics: results will be discussed in Section 3.

\subsection{The sub-resolution model}

The sub-resolution model that we adopt for our cosmological simulations is MUPPI (MUlti Phase Particle Integrator): it represents a multiphase ISM and accounts for a variety of physical processes which occur on scales not explicitly resolved. The model has been introduced and thoroughly described in Murante et al. (2010, 2015) and Valentini et al. (2017, 2019, 2020): in this Section, we outline its main features, while we refer the reader to the aformentioned papers for a more comprehensive discussion and any further details.

\subsubsection{Star formation and stellar feedback}

Our sub-resolution model describes a multiphase ISM. The multiphase particle represents its essential element: it is made up of hot and cold gas in pressure equilibrium, and a possible further stellar component. A gas particle enters a multiphase stage should its density rise above a density threshold $\left(n_{\mathrm{H} \text {, thres }}=0.01 \mathrm{~cm}^{-3}\right)$ and its temperature decrease below a temperature threshold $\left(T_{\text {thresh }}=\right.$ $\left.5 \cdot 10^{4} \mathrm{~K}\right)$

We adopt a set of ordinary differential equations to describe mass and energy flows among different components: radiative cooling makes hot gas condense into a cold phase (whose temperature is fixed to $T_{\mathrm{c}}=300 \mathrm{~K}$ ), while some cold gas evaporates due to the destruction of molecular clouds. We rely on an $\mathrm{H}_{2}$-based SF law to compute the instantaneous SFR of each multiphase particle. A fraction $f_{\mathrm{mol}}$ of the cold gas mass $M_{\mathrm{c}}$ is in the molecular phase: the molecular gas is then converted into stars over a timescale which is the dynamical time of the cold gas $\left(t_{\mathrm{dyn}, \mathrm{c}}\right)$, and according to an efficiency $\left(f_{\star}=0.06\right)$ aimed at capturing the average SF efficiency. Hence, the SFR associated to a multiphase particle reads: $\dot{M}_{\mathrm{sf}}=f_{\star} f_{\text {mol }} M_{\mathrm{c}} / t_{\text {dyn }}$. The molecular fraction $f_{\text {mol }}$ is computed according to the phenomenological prescription by Blitz \& Rosolowsky (2006): $f_{\text {mol }}=1 /\left(1+P_{0} / P\right)$, where $P$ is the hydrodynamic pressure of the gas particle and $P_{0}$ is the pressure of the ISM at which $f_{\mathrm{mol}}=0.5$, this parameter being derived from observations (we adopt a constant value $P_{0} / \mathrm{k}_{\mathrm{B}}=2 \cdot 10^{4} \mathrm{~K} \mathrm{~cm}^{-3}$ ) $\mathrm{SF}$ is implemented according to the stochastic model introduced by Springel \& Hernquist (2003), to spawn stellar particles from gas particles.

Energy contributed by $\mathrm{SN}$ explosions counterbalances radiative cooling, along with the hydrodynamical term accounting for shocks and heating (cooling) due to gravitational compression (expansion) of gas. Stellar feedback releases energy both in thermal and kinetic forms. The thermal stellar feedback energy $\Delta E_{\mathrm{fb}, \text { therm }}=f_{\mathrm{fb} \text {,therm }} E_{\mathrm{SN}} \Delta M_{\star} / M_{\star, \mathrm{SN}}$ is supplied by each multiphase star-forming particle to neighbours within a cone (whose

4 The effective density threshold for the SF is $n_{\text {thresh,sf }} \simeq 66.7 \mathrm{~cm}^{-3}$. Equation $f_{\text {mol }}=1 /\left(1+P_{0} / P\right)$ implies that $n_{\text {thresh,sf }} T_{\mathrm{c}}=2 \cdot 10^{4} \mathrm{~K} \mathrm{~cm}^{-3}$, assuming $n_{\text {thresh,sf }}$ as the number density of the cold gas for which $f_{\mathrm{mol}}=$ 0.5 , and plugging in the adopted value for $P_{0}$. On the other hand, $n_{\text {thres }}$ is the density threshold to let a gas particle sample the multiphase ISM. half-opening angle is $\vartheta=30^{\circ}$ ), in a given time-step. Here, $f_{\mathrm{fb}}$, therm describes the thermal stellar feedback efficiency (i.e. the fraction of $E_{\mathrm{SN}}=10^{51} \mathrm{erg}$ which is actually coupled to the ISM), $M_{\star, \mathrm{SN}}$ is the stellar mass that is required on average to have a single SN II, and $\Delta M_{\star}$ represents the mass of the multiphase particle that has been converted into stars. As for kinetic stellar feedback, which is a key process to drive galactic outflows, our sub-resolution model adopts the galactic outflow model introduced in Valentini et al. (2017). According to this model, the ISM is isotropically provided with kinetic stellar feedback energy. Each star-forming particle supplies the energy: $\Delta E_{\mathrm{fb}, \text { kin }}=f_{\mathrm{fb}, \text { kin }} E_{\mathrm{SN}} \Delta M_{\star} / M_{\star, \mathrm{SN}}$ isotropically, to all the wind particles ${ }^{5}$ within the smoothing length, with kernel-weighted contributions. Here, $f_{\mathrm{fb}}$,kin is the kinetic stellar feedback efficiency (see Section 2.3 for adopted values). Wind particles receiving energy use it to increase their velocity along their least resistance path, since they are kicked against their own density gradient. We refer the interested reader to the aforementioned papers (Murante et al. 2010, 2015; Valentini et al. 2017, 2019) for a thorough description of all the processes included in our sub-resolution model; in particular, Section 2 of Valentini et al. (2020) provides a comprehensive summary and can be taken as a reference for the values of all the parameters not explicitly mentioned here. A gas particle exits the multiphase stage when its density drops below a density threshold $\left(0.2 \rho_{\text {thresh }}\right)$ or once a maximum time (set by the dynamical time of the cold gas) elapses.

MUPPI also features chemical evolution and enrichment, these processes being self-consistently accounted for following the model by Tornatore et al. (2007). Star particles (each describes a simple stellar population, assuming a Chabrier 2003 initial mass function) produce and release heavy elements, which are distributed to neighbouring gas particles with kernel-weighted contributions. We follow the production of heavy elements (13 different metals, plus Hydrogen and Helium) released by aging and exploding stars adopting sets of stellar yields. We assume the stellar yields provided by Thielemann et al. (2003) for SNe Ia and the mass- and metallicity-dependent yields by Karakas (2010) for intermediate and low-mass stars during the AGB phase. As for SNe II, we use the mass- and metallicity-dependent yields by Woosley \& Weaver (1995) and Romano et al. (2010), as detailed in Valentini et al. (2019). Each element separately contributes to the gas cooling rate, that is modelled according to Wiersma et al. (2009). To infer cooling rates, the effect of a spatially uniform, time-dependent ionizing cosmic background (Haardt \& Madau 2001) is accounted for.

\subsubsection{BHs and quasar feedback}

BHs are treated as collisionless sink particles which are seeded in DM haloes whose mass is larger than $M_{\mathrm{DM}}$, seed $=1.48 \cdot 10^{9} \mathrm{M}_{\odot}$ (see below), and which do not already host a BH. A Friends-ofFriends (FoF) algorithm, run on-the-fly, identifies DM haloes. BHs are first introduced with a seed mass $M_{\mathrm{BH}}$, seed $=1.48 \cdot 10^{5} \mathrm{M}_{\odot}$ (similar values for $M_{\mathrm{DM} \text {,seed }}$ and $M_{\mathrm{BH}}$, seed have been previously adopted by e.g. Costa et al. 2014; Barai et al. 2018), in the position of the minimum potential of the halo. Seeding presciptions like the one we use are meant to capture the result of the formation of direct collapse BHs (see Section 1). BHs are pinned to the minimum of the gravitational potential, to prevent them from wandering from the

5 Wind particles are gas particles which sample galactic outflows and are hydrodynamically decoupled from the surrounding medium for a lapse of time (see Valentini et al. 2020, for details). 
centre of the halo in which they reside because of numerical artefacts (Wurster \& Thacker 2013). Hence, at each time-step we shift the BH towards the position of the particle with the absolute minimum value of the local gravitational potential within the gravitational softening of the $\mathrm{BH}$, if not already there (as also done by e.g. Ragone-Figueroa et al. 2013; Schaye et al. 2015; Weinberger et al. 2017; Pillepich et al. 2018a).

Once seeded, BHs grow as a result of two processes: gas accretion and mergers with other BHs. We model gas accretion onto the BH via the Bondi-Hoyle-Lyttleton accretion solution (Hoyle \& Lyttleton 1939; Bondi \& Hoyle 1944; Bondi 1952). The Bondi-like accretion rate is numerically estimated as:

$\dot{M}_{\mathrm{B}}=\frac{4 \pi G^{2} M_{\mathrm{BH}}^{2}\langle\rho\rangle}{\left(\left\langle c_{\mathrm{S}}\right\rangle^{2}+\langle v\rangle^{2}\right)^{3 / 2}}$,

where $G$ is the gravitational constant (Springel et al. 2005). In equation (1), the density of the gas $\langle\rho\rangle$, its sound speed $\left\langle c_{\mathrm{S}}\right\rangle$, and the velocity $\langle v\rangle$ of the $\mathrm{BH}$ relative to the gas are calculated by averaging over SPH quantities of the gas particles within the BH smoothing length, with kernel-weighted contributions. We refer to the smoothing length of a $\mathrm{BH}$ particle by analogy with gas particles, as the radius $h_{\mathrm{i}}$ of the sphere centred on the considered particle, which contains a given number of neighbour particles $(\sim 200$, for the kernel function that we adopt; see Beck et al. 2016, for details). In our simulations, we distinguish between hot and cold gas accretion (see Valentini et al. 2020). We assume a temperature threshold $T_{\text {split }}=5 \cdot 10^{5} \mathrm{~K}$ (e.g. Steinborn et al. 2015) to differentiate hot from cold gas. The accretion rates for the hot and cold phases are estimated separately according to equation (1). Once $\dot{M}_{\mathrm{B}, \mathrm{h}}$ and $\dot{M}_{\mathrm{B}, \mathrm{c}}$ are retrieved, the gas accretion rate $\dot{M}_{\text {accr }}$ is given by the sum of both hot and cold gas accretion, and is limited to the Eddington accretion rate, i.e.:

$\dot{M}_{\mathrm{accr}}=\min \left(\dot{M}_{\mathrm{B}, \mathrm{h}}+\dot{M}_{\mathrm{B}, \mathrm{c}}, \dot{M}_{\mathrm{Edd}}\right)$.

As a result of the gas accretion process, the $\mathrm{BH}$ can absorb neighbour gas particles according to the stochastic scheme by Springel et al. (2005). We do not assume any boost factor in equation (1), neither for the hot nor for the cold gas accretion in our simulations. Indeed, our resolution and sub-resolution modelling of the ISM physics allow us to achieve a fair description of the accretion process and final $\mathrm{BH}$ masses in line with observations without the need for a boost factor (see also Section 4, and the discussion in Sections 1 and 3.2 of Valentini et al. 2020, for further details and caveats).

Moreover, we further improve the modelling of gas accretion by taking into account the angular momentum of the accreting gas (see also Rosas-Guevara et al. 2015; Schaye et al. 2015). We limit the inflow of the cold gas which has a high angular momentum: gas with rotational support is indeed expected to depart significantly from the Bondi assumptions, and prevented from being directly accreted. Therefore, the contribution to the gas accretion rate from the cold gas (entering in equation (2)) is:

$\dot{M}_{\mathrm{B}, \mathrm{c}}=\dot{M}_{\mathrm{B}, \mathrm{c}} \min \left(1, \mathcal{L}_{\mathrm{AM}}\right)$,

where $\mathcal{L}_{\mathrm{AM}}$ is the gas accretion rate limiter, i.e.:

$\mathcal{L}_{\mathrm{AM}}=\frac{1}{C_{\mathrm{visc}}}\left(\frac{c_{\mathrm{s}, \mathrm{c}}}{V_{\phi}}\right)^{3}$.

In equation (4), $C_{\mathrm{visc}}=2 \pi$ is a constant parameter aimed at capturing the viscosity of the accretion disc at the sub-resolution level, $c_{\mathrm{S}, \mathrm{c}}$ is the sound speed of the cold $\left(T<T_{\text {split }}\right)$ gas, and $V_{\phi}$ is the rotational velocity of the cold gas surrounding the $\mathrm{BH}$ (i.e. within its smoothing length; see Valentini et al. 2020, for details). We refer to Valentini et al. (2020) for a thorough investigation into the impact of the angular momentum dependent gas accretion.

The actual mass growth rate of the $\mathrm{BH}$ reads:

$\dot{M}_{\mathrm{BH}}=\left(1-\epsilon_{\mathrm{r}}\right) \dot{M}_{\mathrm{accr}}$.

A small fraction $\epsilon_{\mathrm{r}} \dot{M}_{\text {accr }}$ is radiated away. In the quasar feedback process which ensues from gas accretion, the $\mathrm{BH}$ bolometric luminosity thus reads:

$L_{\mathrm{r}}=\epsilon_{\mathrm{r}} \dot{M}_{\mathrm{accr}} c^{2}=\frac{\epsilon_{\mathrm{r}}}{1-\epsilon_{\mathrm{r}}} \dot{M}_{\mathrm{BH}} c^{2}$.

As for the radiative efficiency, we assume $\epsilon_{\mathrm{r}}=0.03^{6}$ A tiny part of the radiated luminosity $L_{\mathrm{r}}$ is then coupled to the ISM as AGN feedback energy, the feedback energy per unit time being:

$\dot{E}_{\mathrm{fb}, \mathrm{tot}}^{\mathrm{AGN}}=\epsilon_{\mathrm{f}} L_{\mathrm{r}} \approx \epsilon_{\mathrm{f}} \epsilon_{\mathrm{r}} \dot{M}_{\mathrm{BH}} c^{2}$,

where $\epsilon_{\mathrm{f}}$ is the feedback efficiency. We adopt $\epsilon_{\mathrm{f}}=10^{-4}$ : we tuned this efficiency in order to match the normalisation of the black hole to stellar mass relation $\left(M_{\mathrm{BH}}-M_{\star}\right.$, see Section 3.3) at $z=6^{7}$ (Wang et al. 2010; Pensabene et al. 2020). This quasar feedback energy is coupled thermally and isotropically to the $\mathrm{BH}$ neighbouring gas particles.

The quasar feedback energy is distributed to all gas particles within the smoothing sphere of the $\mathrm{BH}$, in a kernel-weighted fashion. Quasar feedback energy contributions assigned to single-phase particles within the BH smoothing volume increase their temperature and specific internal energy. Quasar feedback energy assigned to the multiphase particles within the BH kernel (the vast majority) is distributed to both their hot and cold phases. The covering factors of the hot and cold gas within each multiphase particle determine the fraction of feedback energy provided to the two phases. The physical idea behind this modelling assumes that the larger is the volume occupied by the cold gas, the larger is the amount of energy that it absorbs. The quasar feedback energy coupled to the hot gas within the multiphase particle is used to increase its temperature, while the quasar feedback energy provided to the cold phase produces the evaporation of the cold gas, whose mass is brought to the hot phase. Further details about the modelling of the AGN feeding and feedback processes can be found in Valentini et al. (2020).

The other process contributing to the $\mathrm{BH}$ growth is $\mathrm{BH}-\mathrm{BH}$ merging: two BHs are merged should their distance be smaller than twice their gravitational softening length (the same as $\epsilon_{\mathrm{bar}}$ ), and if their relative velocity $v_{\mathrm{BH}-\mathrm{BH}}<0.5\left\langle c_{\mathrm{s}}\right\rangle$. The resulting $\mathrm{BH}$ lies

6 Such a value for the radiative efficiency is compatible within a factor of 2 with the minimum value of the accretion efficiency for an accretion disc surrounding a non-spinning BH (Shakura \& Sunyaev 1973; Novikov \& Thorne 1973), and is in agreement with results of Sądowski \& Gaspari (2017); Trakhtenbrot et al. (2017b). When low values of $\epsilon_{\mathrm{r}}$ (i.e. non-spinning BHs) are assumed, so that $\epsilon_{\mathrm{r}} /\left(1-\epsilon_{\mathrm{r}}\right) \approx \epsilon_{\mathrm{r}}$ in eq. (6), final results are mainly sensitive to the product $\epsilon_{\mathrm{r}} \epsilon_{\mathrm{f}}$ (see eq. (7)), although a low radiative efficiency always helps the black hole grow faster (see eq. (5)).

7 The value which $\epsilon_{\mathrm{f}}$ is set to in our simulations is lower than commonly adopted values by a factor of $\sim 10$. Values assumed in other works span the range $\sim 10^{-4}-0.15$ (e.g. Ostriker et al. 2010; Dubois et al. 2012; Costa et al. 2014; Lupi et al. 2019; Trebitsch et al. 2020b). Note that sometimes such higher values are calibrated in order to reproduce the normalization of the $M_{\mathrm{BH}}-M_{\star}$ relation in the local universe (e.g. Dubois et al. 2012; Costa et al. 2014), which is below that inferred from observation at $z=6$ by a factor of $\sim 15$ (Wang et al. 2010). When our model is used to reproduce the $M_{\mathrm{BH}}-M_{\star}$ relation at $z=0$, a value $\epsilon_{\mathrm{f}}=0.01$ is adopted, as discussed in Valentini et al. (2020). 


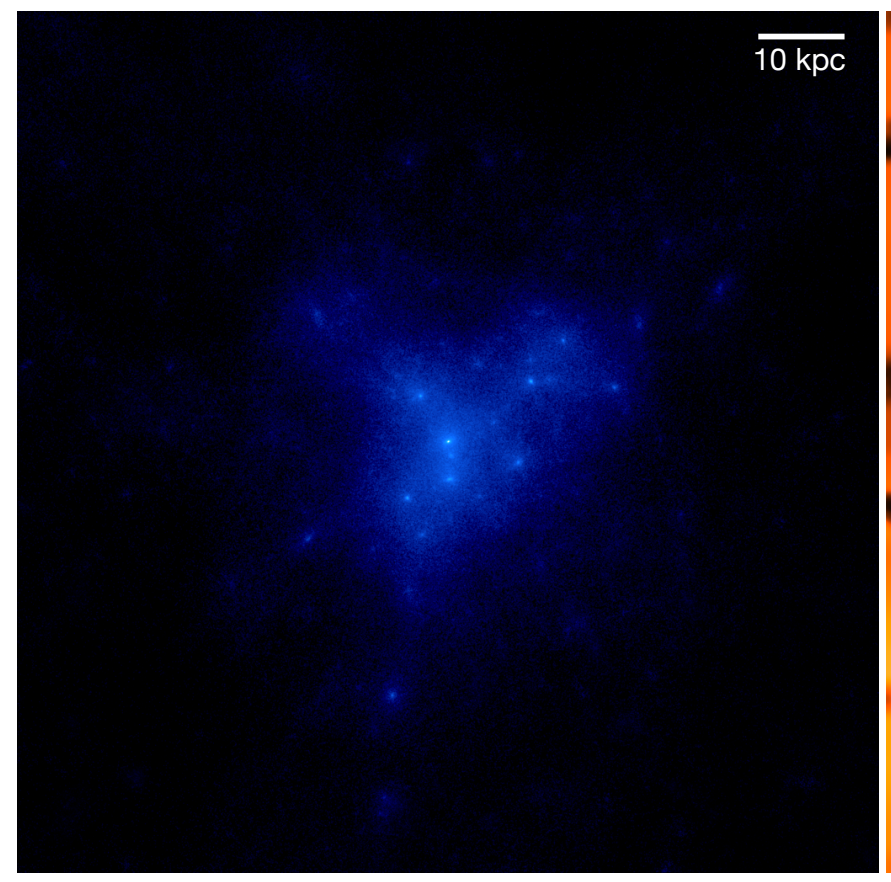

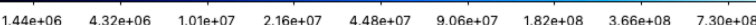

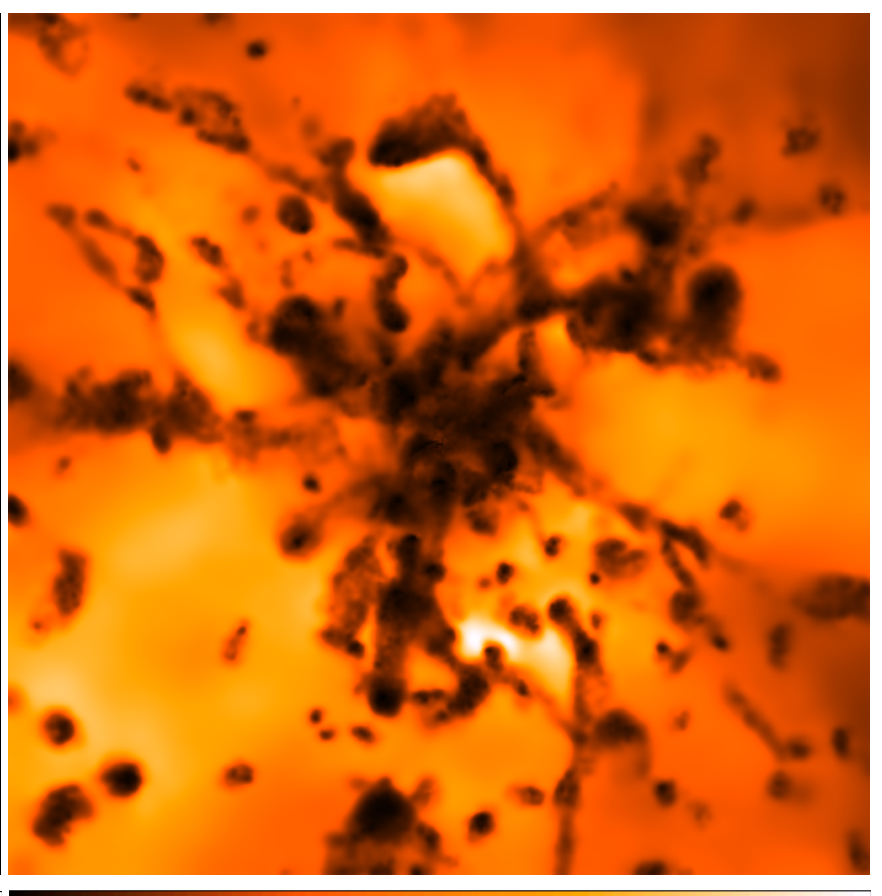

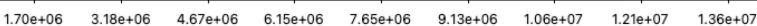

Figure 1. Left panel: Map of total (DM, stars, and gas) mass surface density (in units of $\mathrm{g} / \mathrm{cm}^{2}$ ) of the reference simulation $A G N_{-} f i d$. Right: Projected, smoothed gas temperature map, in units of $\mathrm{K}$. We show a box of $100 \mathrm{pkpc}$ a side at redshift $z=6$, the projection being performed along the $z$-axis (over $100 \mathrm{pkpc}$ ). Both the maps are centred on the centre of the most massive subhalo.

at the position of the most massive one between the two BHs that undergo merging.

\subsection{The set of simulations}

In this work, we show and discuss the results of five different simulations that we carried out. They differ from one another as for the physical processes included or features in the sub-grid modelling. Details are as follows:

- $A G N_{-}$fid is the reference simulation with stellar and quasar feedback. Being the properties of the central BH and its host galaxy (i.e. BH mass and stellar mass of the quasar host; see Section 3.3) in agreement with the $M_{\mathrm{BH}}-M_{\star}$ relation at $z=6$, this is our fiducial model. It includes all the physical processes whose complex interplay we aim at investigating in this work.

- $B H s_{-} n o F B$ is the simulation which includes BHs and the gas accretion process, but no quasar feedback;

- $S F \_o n l y$ is the simulation where BHs are not included and the quasar feedback is not taken into account. It features SF and stellar feedback: the fiducial value $f_{\mathrm{fb}, \text { kin }}=0.12$ for the stellar feedback efficiency is adopted here (see Valentini et al. 2018, 2019), as well as in $A G N_{-}$fid and $B H s_{-} n o F B$;

- $S F \_o n l y \_l o w F B$ is a simulation where BHs and the ensuing feedback are not included, similar to $S F \_o n l y$. In this simulation, a lower kinetic stellar feedback efficiency is assumed with respect to $S F_{\text {_only, i.e. }} f_{\mathrm{fb}, \mathrm{kin}}=0.05$ (the thermal stellar feedback efficiency is always set to $f_{\mathrm{fb} \text {,therm }}=0.2$ ). Should a lower amount of stellar feedback energy be coupled to the ISM in the kinetic form, the resulting outflows are triggered with a lower velocity.

- $A G N \_h i g h F B$ is a simulation analogous to the fiducial $A G N_{-}$fid, but adopts a feedback efficiency $\epsilon_{\mathrm{f}}=10^{-3}$, i.e. higher than the reference value by a factor of 10 . The central, most massive $\mathrm{BH}$ mass of the quasar-host galaxy in this model is in agreement with the $M_{\mathrm{BH}}-M_{\star}$ relation in the local universe (whose normalization is observed to be lower than that at high redshift, see Section 3.3).

This suite of simulations has been designed with the aim to investigate: $i$ ) the relative impact of the SF and quasar feedback processes; $i i$ ) the effect of the AGN feedback on the ISM of the galaxies hosting BHs, and on the growth of the BHs themselves; iii) the possibility that stellar feedback alone is main driver of peculiar observational features, should galactic outflows be launched with different velocities.

Besides the aforementioned simulations that we are going to consider in detail, we also performed several preliminary simulations (see Table B1 in Appendix B), which have been preparatory to this analysis. In particular, they have been fundamental to calibrate AGN feedback efficiencies and to explore the parameter space of our sub-resolution model when used to investigate the high- $z$ universe.

\section{RESULTS}

In this Section, we introduce our results. We will first show (Section 3.1) a broad overview of four simulations among those presented in Section 2.3. Then, we will focus in detail on the properties of the ISM of the quasar-host galaxy (Section 3.2), BHs (Section 3.3), and inflow/outflow (Section 3.4) in the reference simulation $A G N_{-}$fid.

\subsection{Overview of the simulations}

We start our analysis by investigating the final properties of the central galaxy in different simulations, at $z=6$. The central galaxy 

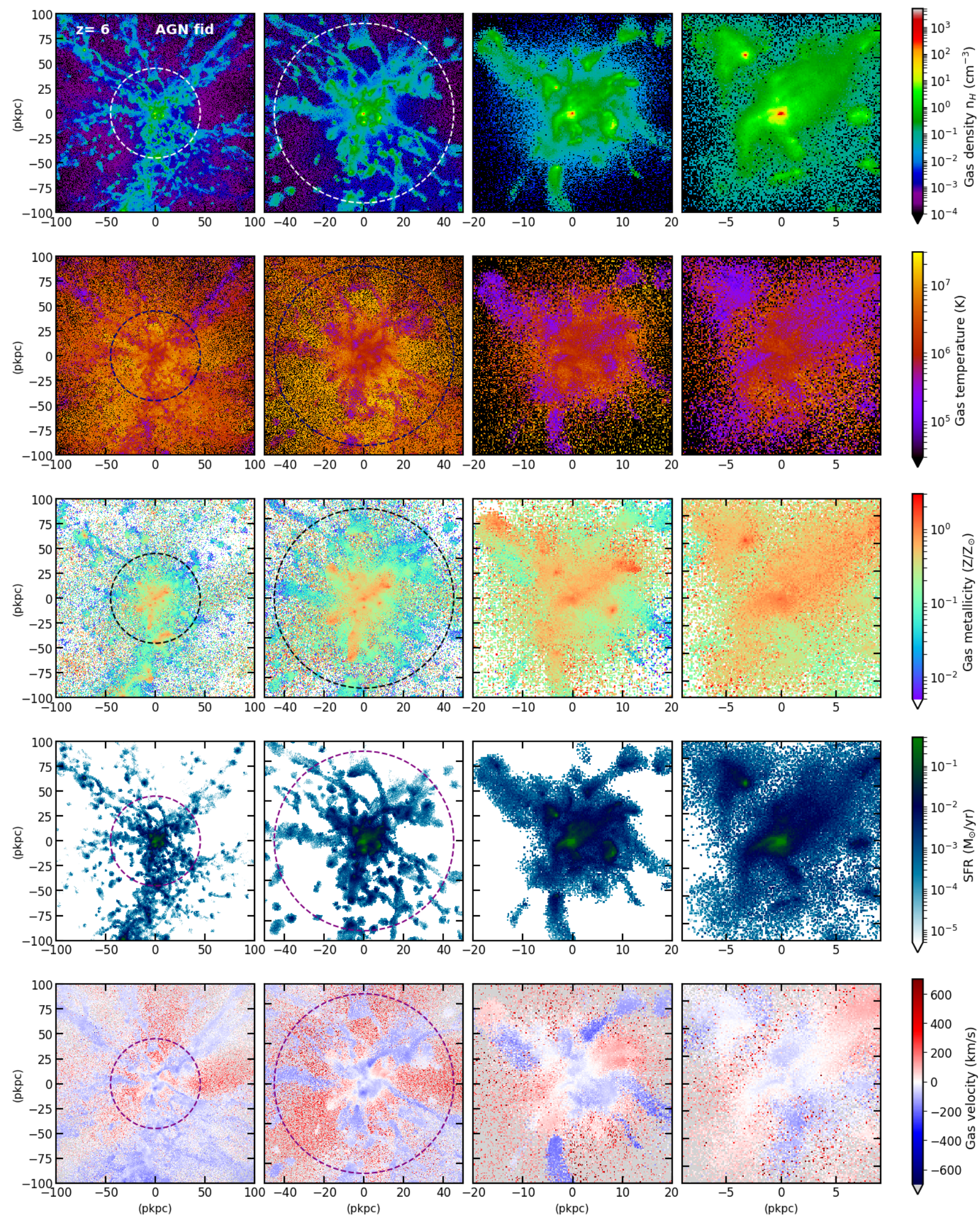

Figure 2. Overview of the fiducial simulation $A G N_{-}$fid, at redshift $z=6$. We show gas density (first row), gas temperature (second row), gas metallicity (third row), the SFR of gas particles (fourth row), and the mass-weighted, radial velocity of gas particles (bottom row). We progressively zoom-in from left to right: the first and second columns show a box of $200 \mathrm{pkpc}$ and $100 \mathrm{pkpc}$ a side, respectively, the projection being performed along the $z$-axis (over 200 pkpc and $100 \mathrm{pkpc}$, respectively). The radius of the dashed circumference shows the virial radius of the central, target halo ( $\mathrm{r}_{\mathrm{vir}}=45.17 \mathrm{pkpc}$ ). The third column shows a box of $40 \mathrm{pkpc}$ (projection is over $20 \mathrm{pkpc}$ along the $z$-axis), while in the fourth column we consider a box of 18 pkpc (projection is over 9 pkpc along the $z$-axis). All the maps are centred on the centre of the most massive subhalo. 

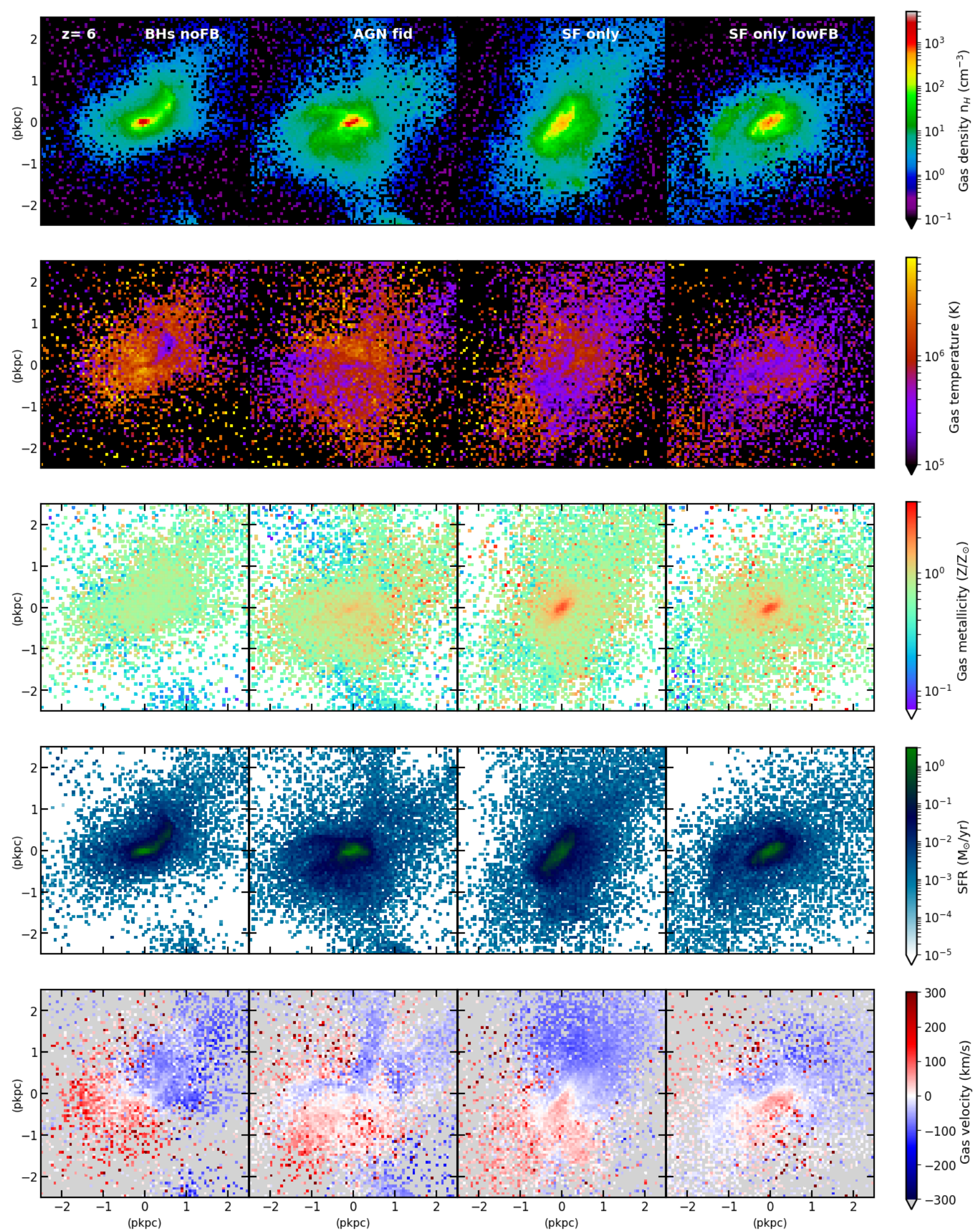

Figure 3. Overview of the four simulated galaxies, at redshift $z=6$. We show gas density (first row), gas temperature (second row), gas metallicity (third row), the SFR of gas particles (fourth row), and the mass-weighted, radial velocity of gas particles (bottom row). Each column considers a different simulation: the first column shows the simulation $B H s_{-}$noFB, the second column depicts $A G N_{-}$fid; the third column shows $S F \_$only, and the fourth column $S F \_o n l y \_l o w F B$. Each box has a side of $5 \mathrm{pkpc}$, and quantities are averaged along the $z$-axis (over $1.2 \mathrm{pkpc}$ ). Bin size is comparable with the softening length of baryonic particles in the simulations. All the maps are centred on the centre of the most massive subhalo. 
is the galaxy located in the most massive sub-halo, which hosts the most massive $\mathrm{BH}$ (should $\mathrm{BHs}$ be present in simulations $A G N_{-}$fid and $\left.B H s \_n o F B\right)$.

Figure 1 introduces our reference simulation $A G N_{-}$fid. We show the projected density of DM, stars, and gas (left panel) and the projected, smoothed, mass-weighted gas temperature map (right panel), at redshift $z=6$. The Figure pictures the connection between the stellar (left panel) and gaseous (right panel) components in the centre of one of our simulated structures. We see clumps and filaments of denser and colder $\left(T \sim 10^{5}-10^{6} \mathrm{~K}\right.$, see also Figure 2) gas, which is mainly inflowing and feeding the central galaxy, surrounded by a hotter and more diffuse phase.

Figure 2 shows a progressive zoom-in on the central galaxy of the reference simulation $A G N_{-}$fid. We analyse gas density, temperature, gas metallicity, the SFR of gas particles, and the massweighted, radial velocity of gas particles with respect to the centre of the target sub-halo. Colours encode mean SPH quantities for gas particles in each spatial bin for all the panels but for those where the SFR is analysed. In this latter case, we consider the sum of the SFR of each gas particle in the bin, to account for the total SFR contributed by the star-forming gas in the bin. As for gas temperature, we consider the SPH estimate for single-phase particle and the mass-weighted average of hot and cold gas temperatures for multiphase particles. We refer to metallicity as overall metal content $Z$, i.e. the total mass of all the elements heavier than Helium that we track in our simulations (see Section 2.2.1) divided by the gas mass, and normalised to the Sun's metallicity. As for the Sun's metallicity, we adopt the present-day value $Z=0.01524$ (Caffau et al. 2011). Gas velocity in each bin is the mass-weighted velocity of gas particles in the bin: as a consequence, in star-forming regions where the bulk of gas is multiphase, the velocity estimate better reflects the velocity of warm and cold gas.

The sequence of panels in Figure 2 shows that the central, quasar-host galaxy is embedded within a complex large-scale structure. It is located in the innermost region of a network of gaseous filaments which bridge surrounding galaxies and sub-structures, and shape the quasar-host galaxy environment. The central galaxy is fed by warm and cold gas which inflows from the large-scale environment. SF mainly occurs in the densest gas knots within the virial radius. The effect of past and ongoing SF is also visible in the distribution of heavy elements: gas metallicity ranges from $\sim 5 \cdot 10^{-3} \mathrm{Z}_{\odot}$ in rarefied gas far from the central galaxy to super-solar values of gas in and around sites of SF. Besides being responsible for metal enrichement of the ISM and circumgalactic medium, stellar feedback also promotes gas to outflow, along with quasar feedback. Radial velocities of the outflowing gas can even exceed $\sim 600 \mathrm{~km} \mathrm{~s}^{-1}$ (see Figure 2 and Section 3.4).

Figure 3 introduces four among the simulations presented in Section 2.3: $B H s_{-} n o F B, A G N \_f i d, S F \_o n l y$, and $S F \_o n l y \_l o w F B$. We show close-up views of the central galaxy in the four different simulations, as the focus of this work is the central galaxy hosted in the target sub-halo, and its ISM. For each simulated galaxy, we analyse gas density, temperature, metallicity, the SFR, and the mass-weighted, radial velocity of gas particles (as in Figure 2). Maps of the galaxy model $A G N_{-}$fid in the second column represent a further zoom-in in the progressive view analysed in Figure 2. We focus on the close-up views in Figure 3 to highlight differences between different models, the larger scale environment being almost indistinguishable among the considered runs (see also Appendix B and Figure B1). Before comparing results from Figures 2 and 3 for different simulations, it is useful to introduce the SFH of the four systems.
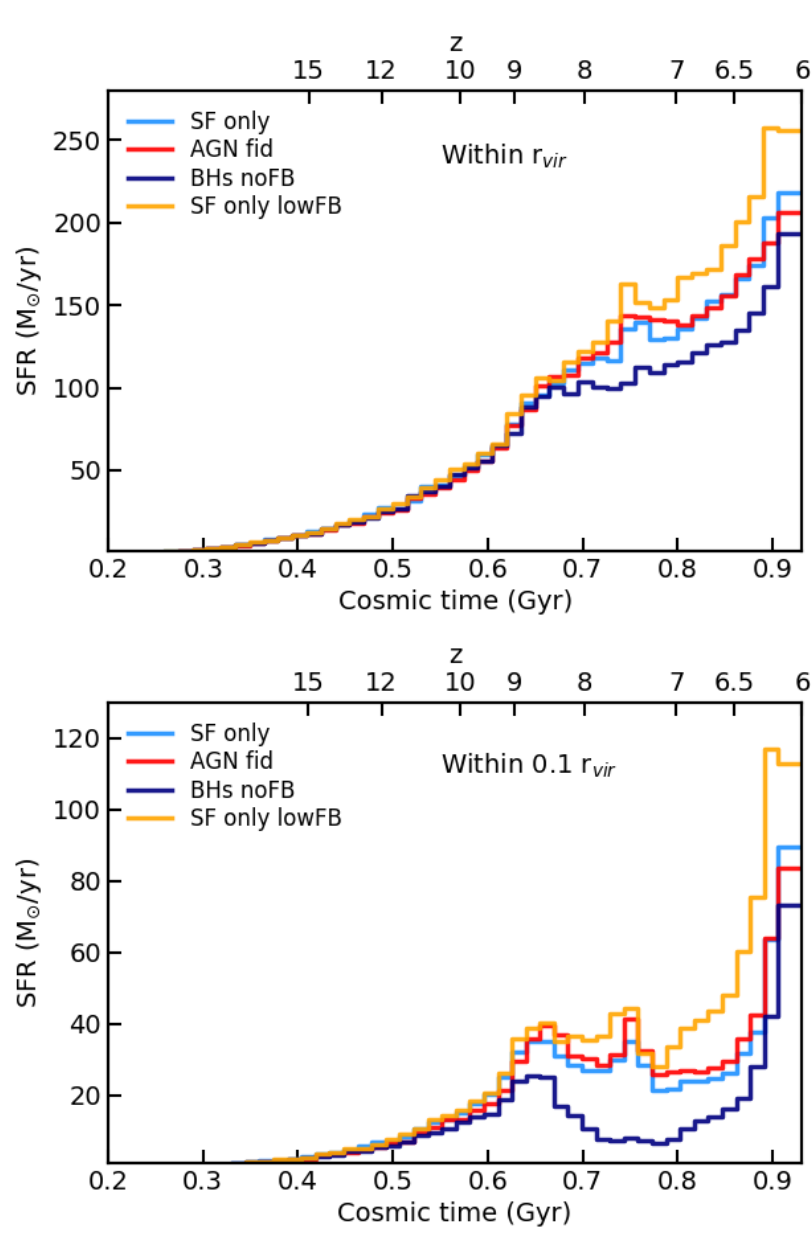

Figure 4. SFHs of the four galaxy models. $S F \_o n l y, A G N \_f i d, B H s \_n o F B$, and $S F \_o n l y \_l o w F B$ are shown in light blue, red, blue, and orange, respectively. SFRs are computed by analysing star particles within the virial radius (top panel) and one tenth of the virial radius (bottom panel) of the most massive sub-halo in each simulation.

In Figure 4 we show the SFH of the four simulated galaxies, within the virial radius and $0.1 \mathrm{r}_{\mathrm{vir}}$ of the central, most massive halo at $z=6$. SFRs are retrieved by analysing stellar age distributions. SFRs of hundreds of $\mathrm{M}_{\odot} \mathrm{yr}^{-1}$ are in good agreement with observations of quasar-host galaxies at $z \sim 6$ (Maiolino et al. 2005; Solomon \& Vanden Bout 2005; Wang et al. 2016; Trakhtenbrot et al. 2017a; Willott et al. 2017; Decarli et al. 2018; Venemans et al. 2018).

All the simulations share a comparable SFH until $z \simeq 10$ is reached. Quasar feedback has only negligible effect on the SFH of the simulated galaxy in our model until $z=6$, and the simulations with $\left(A G N_{-} f i d\right)$ and without (SF_only) AGN feedback share comparable SFRs. The comparison between the two aforementioned models shows that the AGN can have both positive and negative feedback: when the quasar feedback is positive, the SFR can be enhanced because AGN feedback energy overpressurizes the ISM (see Valentini et al. 2020, for details). Episodes of negative quasar feedback (at $z \sim 6$ ) are due to the gas temperature increase induced by $\mathrm{BH}$ feedback on the surrounding gas.

The temperature of the ISM in the galaxy model $A G N_{-}$fid is on average higher than in $S F \_$only (by a factor of $\sim 2$ for distances $r \lesssim 4 \mathrm{kpc}$ from the galaxy centre, as it can be seen by comparing the bottom panels of Figures 6 and B3, and from Figure 3). More- 

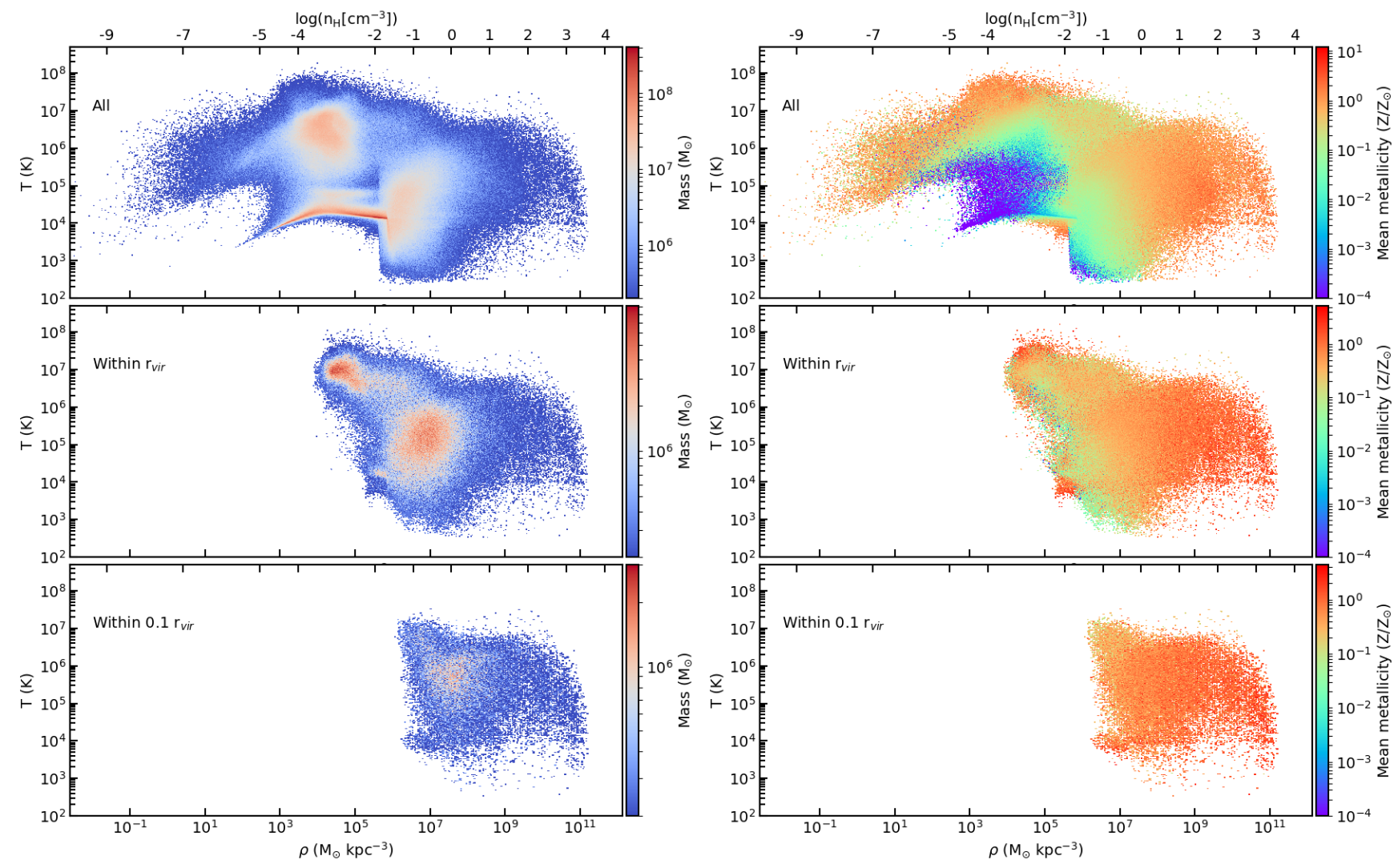

Figure 5. Distribution of gas particles in the density-temperature plane in the reference simulation $A G N_{-} f i d$, at redshift $z=6$. Top panels show the distribution of all the gas particles in the Lagrangian region, middle and bottom panels refer to gas particles within the virial radius $r_{v i r}$ and within $0.1 r_{v i r}$, respectively. The colour encodes the gas mass per density-temperature bin (left-hand panels) and the mean metallicity per bin (right-hand panels). All the color bars in the left set of panels share the minimum value, while the maximum of the color scale is independent for each panel, to better capture features (the same is true for the three panels on the right).

Table 1. Virial radii and stellar masses for different simulations (Column 1) at $z=6$. Column 2: virial radius. Column 3: stellar mass within $\mathrm{r}_{\mathrm{vir}}$. Column 4: stellar mass within $0.1 \mathrm{r}_{\mathrm{vir}}$.

\begin{tabular}{lccc}
\hline Simulation & $\begin{array}{c}\mathrm{r}_{\mathrm{vir}} \\
(\mathrm{pkpc})\end{array}$ & $\begin{array}{c}M_{*}\left(r<\mathrm{r}_{\mathrm{vir}}\right) \\
\left(10^{10} \mathrm{M}_{\odot}\right)\end{array}$ & $\begin{array}{c}M_{*}\left(r<0.1 \mathrm{r}_{\mathrm{vir}}\right) \\
\left(10^{10} \mathrm{M}_{\odot}\right)\end{array}$ \\
\hline \hline AGN_fid & 45.17 & 4.03 & 1.13 \\
\hline BHs_noFB & 44.95 & 3.51 & 0.68 \\
\hline SF_only & 44.64 & 4.01 & 1.07 \\
\hline SF_only_lowFB & 44.68 & 4.55 & 1.44 \\
\hline \hline
\end{tabular}

over, the central region of the galaxy $S F \_o n l y$ is more enriched in heavy metal $\left(\gtrsim 3 \mathrm{Z} / \mathrm{Z}_{\odot}\right.$, while the ISM has a solar metallicity in the innermost regions of the galaxy $A G N_{-}$fid, see Figure 3), as a consequence of the higher SFR at $z=6$. Gas metallicities close to solar or super-solar in the innermost regions of high-redshift quasar-host galaxies are in agreement with observations (e.g. Juarez et al. 2009; Tang et al. 2019; Venemans et al. 2017c, and references therein).

As for the gas velocity in $A G N \_f i d$ and $S F \_o n l y$, Figure 3 shows that the quasar feedback is responsible for promoting outflowing gas to higher velocities (see also Section 3.4). Also, the highest velocity gas outflowing from the innermost region of the quasar-host galaxy $A G N \_f i d$ has a more bipolar geometry with respect to gas outflowing in $S F \_$only, the latter not showing a definite pattern. An enhanced bipolarity in the $A G N_{-} f i d$ model is due to the additional energy source represented by the central AGN. Interestingly, we find that the presence of the AGN is also responsible for disarranging the gas motion in the innermost regions of the galaxy (gas kinematics being less disturbed in $S F_{-}$only than in $A G N_{-} f i d$ ).

When the central $\mathrm{BH}$ only accretes gas without injecting quasar feedback energy in the ISM $\left(B H s \_n o F B\right)$, the SFR is lower with respect to all the other models. In this case, the central $\mathrm{BH}$ accretes more gas and grows way more massive with respect to the reference $A G N_{-}$fid model (see Section 3.3): as a consequence, the central regions of the quasar-host galaxy lack fuel for SF as the gas is mainly accreted by the SMBH. The lower SFR in this model also stems from the higher temperature of the ISM (Figure 3), and reflects on the lower gas metallicity.

The model SF_only_lowFB has the highest SFRs: when a lower kinetic stellar feedback efficiency is adopted (with respect to $S F \_o n l y$, see Section 2.3), the velocity of particles receiving stellar feedback energy is boosted to lower values (see also Section 3.4). Thus, a lower amount of gas is pushed far from sites of SF, and a larger reservoir of gas keeps fuelling SF in the galaxy. This galaxy model is also characterised by a higher gas metallicity, due to the higher SFR at $z \sim 6$.

Virial radii of the central sub-halo in the four models, as well as the stellar mass enclosed within the virial radius and $0.1 \mathrm{r}_{\mathrm{vir}}$ are listed in Table 1. Stellar masses of few $10^{10} \mathrm{M}_{\odot}$ in haloes as massive 
as $\sim 10^{12} \mathrm{M}_{\odot}$ at $z=6$ are also in line with predictions from stellarto-halo mass relations obtained via abundance matching techniques (Behroozi et al. 2013).

\subsection{The host galaxy and its ISM}

In this Section, we analyse the main features of the ISM of the quasar-host galaxy in our fiducial model.

Figure 5 shows the mass and metallicity distribution in the density-temperature phase diagram of gas particles in the simulation $A G N_{-} f i d$, at redshift $z=6$. The density of gas particles in Figure 5 is the SPH density; the temperature of gas particles is the SPH estimate for single-phase particles and the mass-weighted average of the temperatures of the hot and cold phases for multiphase particles. Multiphase particles in Figure 5 occupy the region where $\log \left(n_{\mathrm{H}}\left[\mathrm{cm}^{-3}\right]\right)>-2$ (corresponding to $n_{\mathrm{H}}$, thres; see Section 2.2.1). They scatter across an area that spans more than four orders of magnitude both in density and in temperature: such a spread is a characteristic feature of the advanced modelling of the ISM in our sub-resolution model. Indeed, the MUPPI sub-resolution model follows the dynamical evolution of the ISM and considers that the average energy of multiphase gas depends on its past history (the solution of the equations describing the ISM is not obtained under an equilibrium hypothesis). On the other hand, the spread in density and temperature would not be present if multiphase particles obeyed an equation of state (e.g. Springel \& Hernquist 2003), where the pressure $P$ of multiphase particles is a function of their density through a polytropic equation for $P(\rho)$ (see discussion in Valentini et al. 2017, for details).

The right panels of Figure 5 show that the metallicity of gas spans more than five orders of magnitude, ranging from supersolar metallicity down to $\sim 10^{-4} \mathrm{Z}_{\odot}$. Extremely metal-poor gas is mainly warm $\left(\sim 5 \cdot 10^{3}<T[K]<10^{6}\right)$ and rarefied $(-6<$ $\left.\log \left(n_{\mathrm{H}}\left[\mathrm{cm}^{-3}\right]\right)<-2\right)$. The ISM within the innermost region of the main galaxy (bottom right panel), on the other hand, has been significantly enriched by stellar evolution: its metallicity is rather homogeneous, and ranges from slightly sub-solar to super-solar.

Figure 6 shows radial profiles within twice the virial radius of the most massive subhalo in the reference simulation $A G N_{-} f i d$, at redshift $z=6$. In the top panel, we analyse density profiles of gas, stars, DM, and baryons, while the middle and bottom panels illustrate gas number density and mass-weighted temperature, respectively. This figure provides complementary information to Figure 5, showing that the densest and coldest gas is located in the innermost regions of the quasar-host galaxy. The mean gas temperature increases from few $10^{5} \mathrm{~K}$ in the centre to $\sim 4 \cdot 10^{6} \mathrm{~K}$ at the virial radius, and then it mildly declines. The profile is not smooth, due to the presence of substructures and clumps, as it can be seen from Figure 2 and especially from Figure 1 (right panel). These colder clumps are also responsible for the temperature decrease beyond the virial radius (see also Figure 2, second row).

Figure 7 shows the time evolution of the mass of gas, stars, and metals within the virial radius and $0.1 \mathrm{r}_{\mathrm{vir}}$ of the most massive subhalo in the fiducial simulation $A G N \_f i d$, at $z=6$. In the figure we analyse the total amount of gas (contributed by all the gas particles within either $r_{v i r}$ or $0.1 r_{v i r}$ ), the mass of cold gas (which constitutes the bulk of the mass of multiphase gas particles; see Section 2.2.1), and the molecular gas mass (representing a fraction of the mass of cold gas, as detailed in Section 2.2.1). We also consider the mass of metals (contributed by all the heavy elements within gas particles), and the stellar mass. The mass of gas (and thus that of metals and stars) smoothly increases across the (almost) entire time frame, as

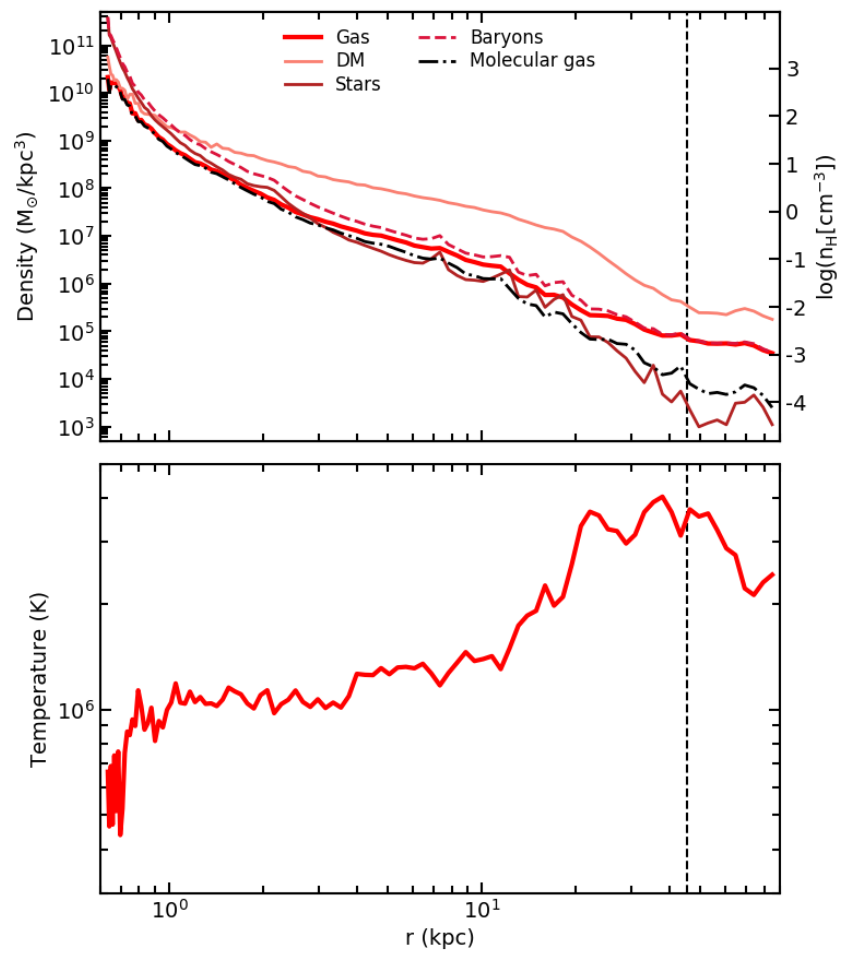

Figure 6. Density and temperature radial profiles within twice the virial radius in the reference simulation $A G N_{-} f i d$, at redshift $z=6$. We show the density profile of gas (total and molecular), stars, DM, and baryons (top panel), and the mass-weighted, temperature profile (bottom panel). The vertical, dashed black line highligths the virial radius of the most massive subhalo.

Table 2. Relevant masses of the quasar-host galaxy $A G N_{-} f i d$ within a given distance (Column 1) from the centre. Column 2: total mass of gas. Column 3: mass of cold gas. Hot gas mass is $M_{\text {hot }}=M_{\text {gas }}-M_{\text {cold }}$. Column 4: mass of molecular gas. Column 5: mass of metals in the gaseous phase. Column 6: stellar mass.

\begin{tabular}{lccccc}
\hline $\begin{array}{l}\text { Simulation } \\
A G N_{-} f i d \\
\text { at } z=6\end{array}$ & $\begin{array}{c}M_{\text {gas }} \\
\left(10^{10} \mathrm{M}_{\odot}\right)\end{array}$ & $\begin{array}{c}M_{\text {cold }} \\
\left(10^{10} \mathrm{M}_{\odot}\right)\end{array}$ & $\begin{array}{c}M_{\text {mol }} \\
\left(10^{10} \mathrm{M}_{\odot}\right)\end{array}$ & $\begin{array}{c}M_{\text {metals }} \\
\left(10^{8} \mathrm{M}_{\odot}\right)\end{array}$ & $\begin{array}{c}M_{*} \\
\left(10^{10} \mathrm{M}_{\odot}\right)\end{array}$ \\
\hline \hline within $\mathrm{r}_{\mathrm{vir}}$ & 8.05 & 4.91 & 3.32 & 4.26 & 4.03 \\
\hline within $0.1 \mathrm{r}_{\mathrm{vir}}$ & 0.82 & 0.68 & 0.64 & 0.86 & 1.13 \\
\hline \hline
\end{tabular}

a consequence of the gas which is accreted from the large scale structure and which provides the reservoir for SF. Focussing on the evolution within $0.1 \mathrm{r}_{\text {vir }}$ (bottom panel) at $9>z>8.5$, it is possible to see that the amount of gas decreases, as a consequence of the gas expelled beyond $0.1 r_{\text {vir }}$ by galactic outflows triggered by ongoing SF (see Figure 4).

Figure 7 also illustrates how significant the contribution of the cold and molecular phases is to the total amount of gas, especially within $0.1 \mathrm{r}_{\mathrm{vir}}$. When the ISM is almost entirely multiphase, the cold gas accounts for $\sim 85 \%$ of the total gas, while the hot phase contributes little. As for the amount of cold gas which is in the molecular phase, it depends on the ISM properties (i.e. gas pressure and thus density) through the molecular fraction $f_{\text {mol }}$ (see Section 2.2.1). While this fraction spans the entire range of values when considering multiphase gas within the virial radius, it eas- 


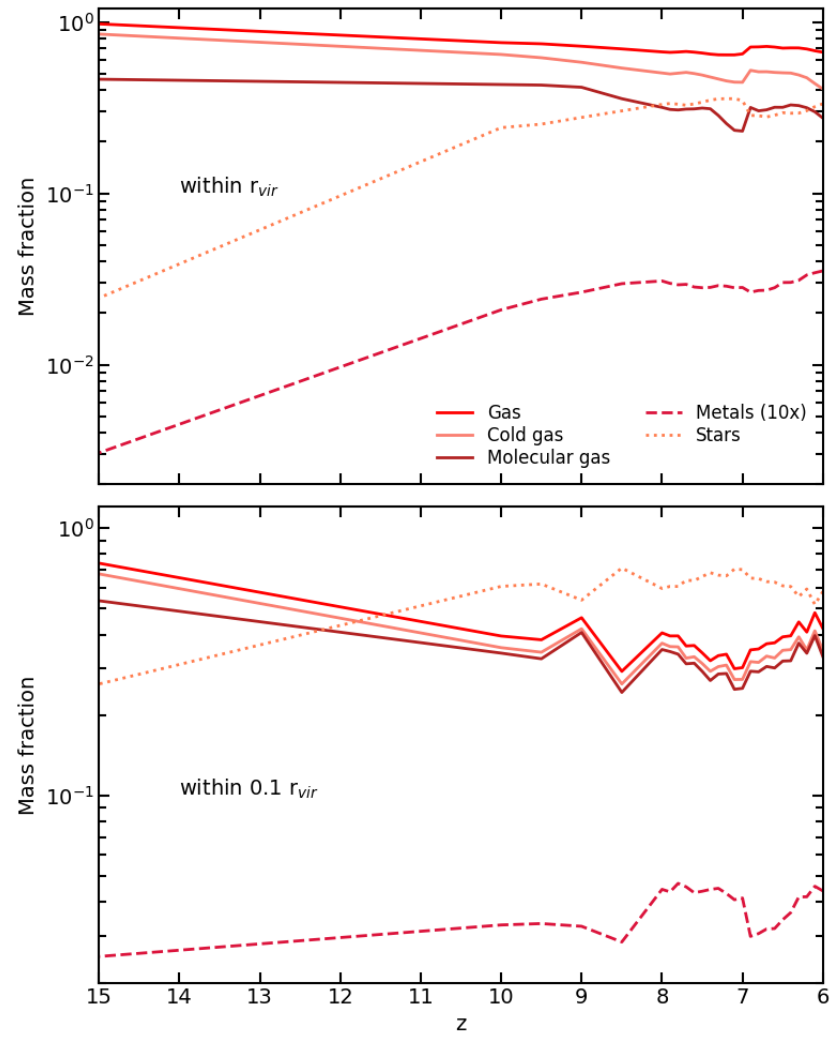

Figure 7. Time evolution of gas, stellar, and metal mass within the virial radius (top panel) and within $0.1 \mathrm{r}_{\mathrm{vir}}$ (bottom panel) in the reference simulation $A G N_{-} f i d$, down to redshift $z=6$. We show the mass fraction (normalised to the total baryonic mass) of all the gas, the cold gas $(T=300 \mathrm{~K})$, the molecular gas, stars, and metals (multiplied by a factor of 10).

Table 3. Most massive $\mathrm{BH}$ and subhalo properties for different simulations (Column 1) at $z=6$. Column 2: most massive, central BH mass. Columns 3 and 4: most massive $\mathrm{BH}$ accretion rate, in units of $\mathrm{M}_{\odot} / \mathrm{yr}$ and Eddington accretion rate, respectively. Column 5: stellar mass of the subhalo which hosts the most massive BH (as identified by the SUBFIND algorithm).

\begin{tabular}{lcccc}
\hline Simulation & $\begin{array}{c}M_{\mathrm{BH}} \\
\left(\mathrm{M}_{\odot}\right)\end{array}$ & $\begin{array}{c}\dot{M}_{\mathrm{BH}} \\
\left(\mathrm{M}_{\odot} / \mathrm{yr}\right)\end{array}$ & $\begin{array}{c}M_{*} \\
\left(\dot{M}_{\mathrm{Edd}}\right)\end{array}$ & $\left(10^{10} \mathrm{M}_{\odot}\right)$ \\
\hline \hline AGN_fid & $9.85 \cdot 10^{8}$ & 35.53 & 0.495 & 2.63 \\
\hline BHs_noFB & $4.62 \cdot 10^{11}$ & $3.17 \cdot 10^{4}$ & 0.978 & 2.26 \\
\hline AGN_highFB & $4.16 \cdot 10^{7}$ & $8.53 \cdot 10^{-2}$ & 0.028 & 2.64 \\
\hline \hline
\end{tabular}

ily approaches $\sim 0.8-1$ (for the majority of gas particles) within $0.1 \mathrm{r}_{\mathrm{vir}}$, where the ISM is denser and more pressurised due to the activity of AGN and especially stellar feedback. As a consequence, the mass of molecular gas is close to that of the cold gas. Masses of gas (total, cold, and molecular), metals and stars within the virial radius and $0.1 \mathrm{r}_{\mathrm{vir}}$ of the quasar-host galaxy $A G N_{-} f i d$ at $z=6$ are listed in Table 2.

\subsection{BH properties}

In this Section, we discuss the properties of $\mathrm{BHs}$ in our reference simulation. Figure 8 introduces the distribution of all the BHs in the
Table 4. Main features of the 2 most accreting BHs after the central, most massive one (columns 2 and 3 ) and of the two closest BHs to the most massive one (columns 4 and 5) in the simulation $A G N_{-}$fid at $z=6$. Row 1 : mass. Row 2: accretion rate. Row 3: distance from the most massive $\mathrm{BH}$.

\begin{tabular}{lcccc}
\hline Simulation & \multicolumn{2}{c}{ Most accreting BHs } & \multicolumn{2}{c}{ Closest BHs } \\
AGN_fid $(z=6)$ & 2nd & 3rd & 1st & 2nd \\
\hline \hline$M_{\mathrm{BH}}\left(\mathrm{M}_{\odot}\right)$ & $9.34 \cdot 10^{6}$ & $2.71 \cdot 10^{8}$ & $1.48 \cdot 10^{5}$ & $1.48 \cdot 10^{5}$ \\
\hline$\dot{M}_{\mathrm{BH}}\left(\mathrm{M}_{\odot} / \mathrm{yr}\right)$ & 0.66 & 0.55 & $5.5 \cdot 10^{-7}$ & $2.7 \cdot 10^{-7}$ \\
\hline $\mathrm{d}(\mathrm{pkpc})$ & 6.87 & 105.4 & 0.023 & 0.024 \\
\hline \hline
\end{tabular}

reference simulation $A G N_{-}$fid at $z=6$, along with gas and stars. $\mathrm{BHs}$ are color-coded according to their accretion rate, while the size of each circle scales with the $\mathrm{BH}$ mass. $\mathrm{BH}$ masses range from the adopted seed value $\left(M_{\mathrm{BH}}\right.$, seed $=1.48 \cdot 10^{5} \mathrm{M}_{\odot}$, see Section 2.2.2) to the most massive $\mathrm{BH}$ formed $M_{\mathrm{BH}}=9.85 \cdot 10^{8} \mathrm{M}_{\odot}$. There are 2 BHs more massive than $10^{8} \mathrm{M}_{\odot}$ in the simulation $A G N_{-}$fid, $3 \mathrm{BHs}$ whose mass is in the range $10^{7}-10^{8} \mathrm{M}_{\odot}$, and $9 \mathrm{BHs}$ with mass between $10^{6}$ and $10^{7} \mathrm{M}_{\odot}$. The accretion rate of the most massive $\mathrm{BH}$ is $\dot{M}_{\mathrm{BH}}=35.53 \mathrm{M}_{\odot} / \mathrm{yr}$ (see Table 3). The most massive $\mathrm{BH}$ was seeded at $z=12.53$ and has since then experienced 30 mergers with other BHs. The last 8 mergers experienced occurred between $6.1<z<6$. The main properties of the two most accreting BHs after the central, most massive one and those of the two closest BHs to the most massive one at $z=6$ are listed in Table 4. The two closest BHs are BHs which have just been seeded.

\subsubsection{SMBH mass growth}

Figure 9 shows the $\mathrm{BH}$ mass growth as a function of the redshift for the central, most massive $\mathrm{BH}$ in three simulations of our suite. Besides $A G N_{-}$fid and $B H s_{-} \_$noFB, here we also consider results from the simulation $A G N \_h i g h F B$. This simulation (see Section 2.3 and Table B1) is analogous to the fiducial $A G N_{-}$fid but adopts a feedback efficiency higher ${ }^{8}$ than the reference value by a factor of 10 .

Figure 9 also quantifies the contributions to $\mathrm{BH}$ mass growth from the two possible channels, namely gas accretion and mergers with other BHs. The shaded region underlying each of the three curves shows the $\mathrm{BH}$ mass increase due to $\mathrm{BH}$ mergers. Thus, the lower border of the shaded area shows the mass that the $\mathrm{BH}$ would have if it only grew because of gas accretion. By analysing the shaded area we can thus appreciate the marginal contribution of $\mathrm{BH}-\mathrm{BH}$ merger to the increase of $\mathrm{BH}$ mass. This negligible contribution mainly stems from the fact that the central SMBH experiences mergers with BHs whose mass is by far smaller than its own.

Table 3 lists mass and accretion rate of the central $\mathrm{BH}$ in $B H s \_n o F B$. Although the AGN feedback in our model does not impact significantly on the physical properties of the ISM of the quasar-host galaxy, it has a key role in regulating the SMBH mass

${ }^{8}$ We further note that the simulation $A G N \_h i g h F B$ adopts a feedback efficiency $\left(\epsilon_{\mathrm{f}}=0.001\right)$ that is ten times lower than the reference model of Valentini et al. 2020 (i.e. $\epsilon_{\mathrm{f}}=0.01$ ). This is because, while in the latter simulation BHs have more than $13 \mathrm{Gyr}$ to grow supermassive, in the former they have less than 1 Gyr to reach masses that are in agreement with observations in the local universe. As a consequence, a weaker AGN feedback is required. 

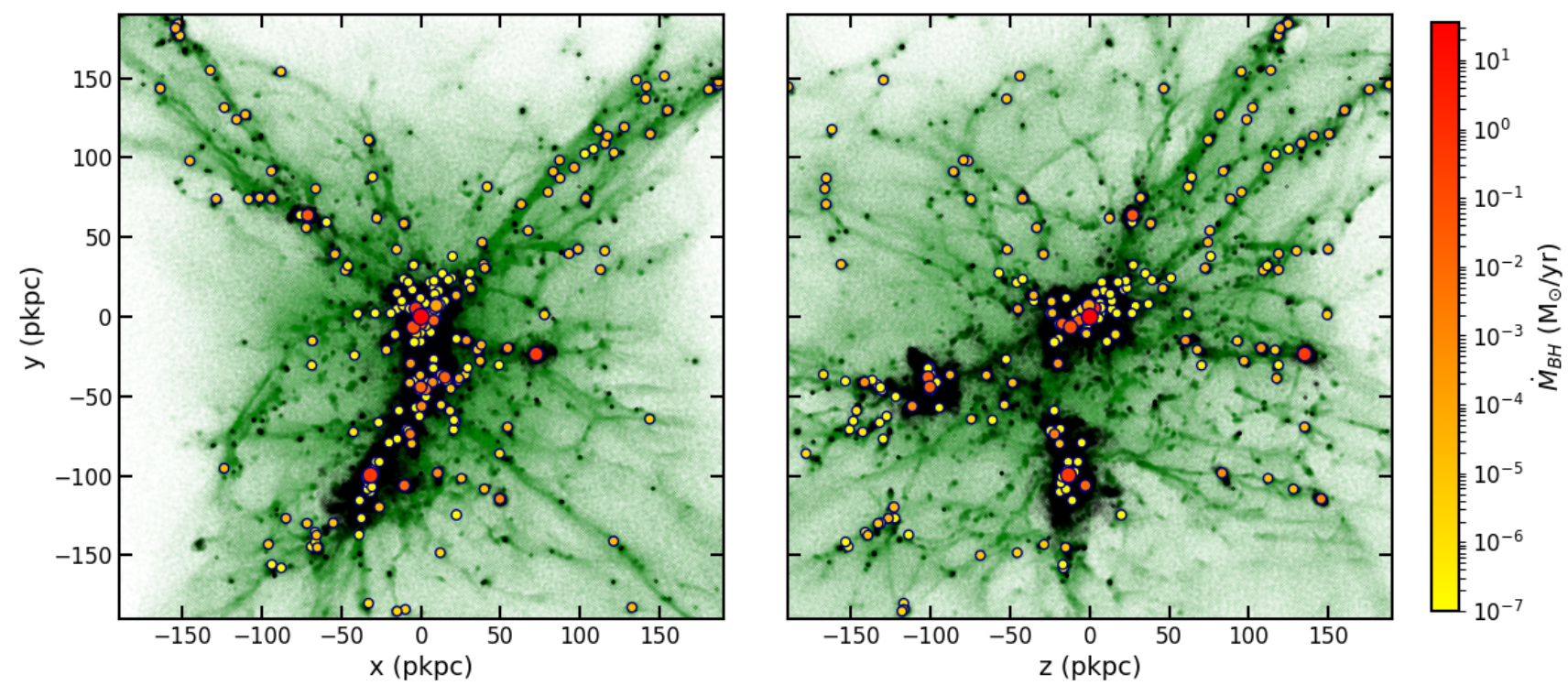

Figure 8. Projected distribution of all the BHs in the reference simulation $A G N_{-}$fid at redshift $z=6$, in the planes $x-y$ (left-hand panel) and $z-y$ (right-hand panel). The colour bar encodes the BH accretion rate. The size of each circle scales with the BH mass. Distances are expressed in physical kpc (pkpc), with respect to the position of the most massive $\mathrm{BH}$, which resides at the centre of the most massive subhalo. Gas (green) and stellar (black) particle distributions are overlaid.

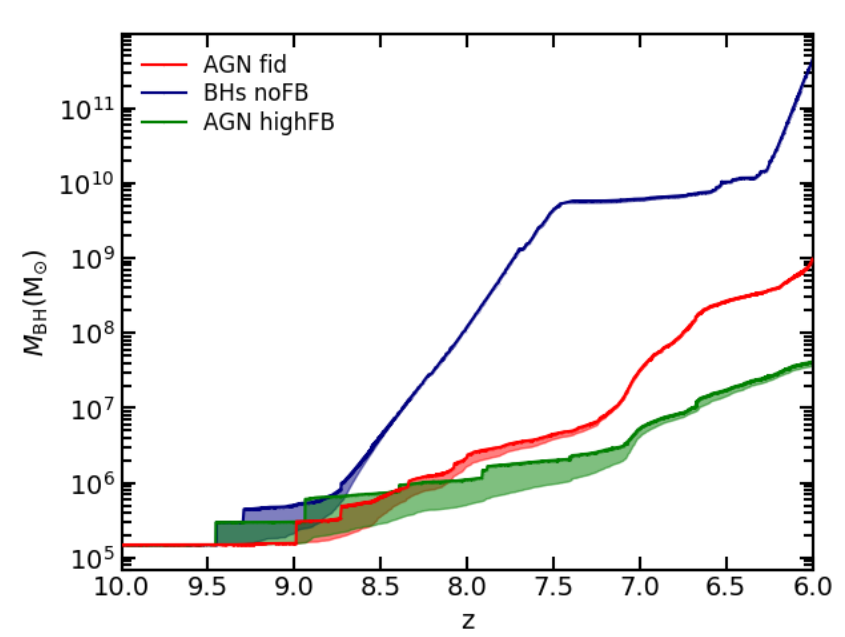

Figure 9. Time evolution of the $\mathrm{BH}$ mass for the central, most massive $\mathrm{BH}$ in the simulations $A G N \_f i d$ (red curve), $B H s \_n o F B$ (blue), and $A G N \_h i g h F B$ (green). The shaded region for each curve shows the contribution to $\mathrm{BH}$ mass growth from mergers with other BHs, which is negligible with respect to gas accretion, at $z=6$. The final mass of the $\mathrm{BH}$ in $A G N_{-}$fid is in agreement with observations at $z=6$ (see Figure 11), while the final mass of the model $A G N \_h i g h F B$ is in line with the $M_{\mathrm{BH}}-M_{\star}$ relation observed at low redshift.

growth. Albeit a tiny amount $\left(\epsilon_{\mathrm{r}} \cdot \epsilon_{\mathrm{f}}=3 \cdot 10^{-6}\right)$ of the rest-mass energy that the $\mathrm{BH}$ accretes is coupled to the ISM, this AGN feedback energy is crucial to avoid that the SMBH grows way too massive (the inclusion of AGN feedback reduces the final $\mathrm{BH}$ mass by a factor of $\sim 400$ ).

Main features of the central, most massive BH in $A G N \_h i g h F B$ and its host subhalo are listed in Table 3, and are in agreement with the $M_{\mathrm{BH}}-M_{\star}$ relation inferred from low-redshift observa- tions (see below). By adopting the following relation ${ }^{9} M_{\mathrm{UV}}=$ $-21.7-2.5 \log _{10} \dot{M}_{\mathrm{BH}}$ to estimate the intrinsic, dust unabsorbed UV magnitude of the quasar as a function of the $\mathrm{BH}$ accretion rate (in units of $\mathrm{M}_{\odot} / \mathrm{yr}$ ), we obtain $M_{\mathrm{UV}}=-25.6$ for the most massive BH in $A G N \_$fid at $z=6$, while $M_{\mathrm{UV}}=-19$ in $A G N \_h i g h F B$ (see Figure 10, also to appreciate how variable $\mathrm{BH}$ accretion rates and hence AGN luminosities are). This low luminosity for the SMBH in $A G N \_h i g h F B$ (that would not be in agreement with the luminosity of the quasar sample by Matsuoka et al. 2018) supports the need to calibrate $\mathrm{BH}$ physics according to high-redshift observations (see Sections 3.3.2 and 3.3.3) to study high-redshift quasars in simulations.

As for the role played by the adopted angular momentum dependent gas accretion onto SMBHs in determining final BH masses, we have thoroughly investigated this process in Valentini et al. (2020). We find that when the accretion of cold gas that is supported by rotational velocity is diminished via equation (3), the evolution of the $\mathrm{BH}$ mass changes: reducing the accretion of cold gas delays and decreases the $\mathrm{BH}$ growth. The higher the values of $C_{\text {visc }}$ that are adopted (equation (4)), the more significant is the BH growth reduction. The impact of different values of $C_{\text {visc }}$ is thoroughly quantified in Valentini et al. (2020) (see in particular Section 5.6, and Figures 16 and 17 of that paper).

\subsubsection{SMBH accretion rates}

The evolutions of the most massive $\mathrm{BH}$ accretion rate in $A G N_{-}$fid and $A G N \_h i g h F B$ are presented in Figure 10. The top panel describes the redshift evolution of the accretion rate in units of $\mathrm{M}_{\odot} / \mathrm{yr}$, while the bottom panel shows the same evolution in units of the

\footnotetext{
9 We adapted the relation from Di Mascia et al. (2021a) by considering the value of the radiative efficiency adopted in our simulations $\left(\epsilon_{\mathrm{r}}=0.03\right.$ instead of the commonly assumed 0.1 ).
} 

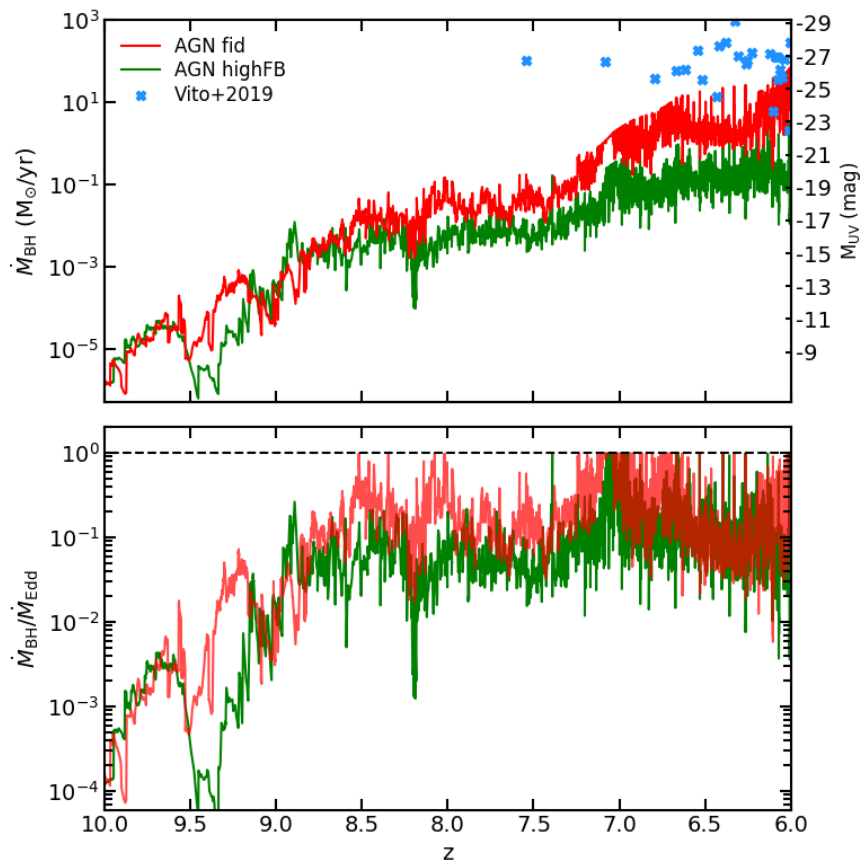

Figure 10. Evolution of the accretion rate of the most massive BH in the simulations $A G N_{-} f i d$ and $A G N \_h i g h F B$. The same evolution is shown both in units of $\mathrm{M}_{\odot} / \mathrm{yr}$ (top panel) and in units of the Eddington accretion rate (bottom panel). The dashed black line where $\dot{M}_{\mathrm{BH}} / \dot{M}_{\mathrm{Edd}}=1$ marks the maximum allowed $\mathrm{BH}$ accretion rate in our models. Observational data from Vito et al. (2019).

Eddington accretion rate. The $\mathrm{BH}$ accretion rate is capped to the Eddington accretion rate (dashed line in Figure 10; see Section 2.2.2) in our simulations. We also include observational data from the high-z quasar sample of Vito et al. (2019): we converted observed UV magnitudes in $\mathrm{BH}$ accretion rates by exploiting the same relation used in Section 3.3.1. The top panel of Figure 10 shows how the accretion rate increases as the redshift decreases; by focussing on the bottom panel, it is possible to see that the two BHs experience an early phase $(z \gtrsim 9)$ of low-accretion rate and then they enter a higher-accretion rate stage. The commonly adopted threshold to distinguish between high- and low-accretion rate mode feedback is $\dot{M}_{\mathrm{BH}} / \dot{M}_{\mathrm{Edd}}=10^{-2}$ (e.g. Churazov et al. 2005; Sijacki et al. 2007). For redshifts lower than $z \sim 9$, the most massive BH in $A G N_{-}$fid always accretes at high-accretion rates (quasar mode). In particular, the SMBH is characterised by several episodes where its accretion is Eddington limited. Throughout the $\mathrm{BH}$ evolution, the accretion of cold gas always dominates over the accretion of the hot gas, and it almost amounts to the total $\mathrm{BH}$ accretion rate (see equation (2)). At $z=6$, the accretion rate (in units of the Eddington accretion rate) of the most massive $\mathrm{BH}$ in $A G N_{-}$fid is $\dot{M}_{\mathrm{BH}} / \dot{M}_{\mathrm{Edd}}=0.495$. This value is significantly lower for the central BH in $A G N \_h i g h F B$, where $\dot{M}_{\mathrm{BH}} / \dot{M}_{\mathrm{Edd}}=2.77 \cdot 10^{-2}$ highlights an AGN activity which is at the limit of the quasar phase (according to the aforementioned criterion). There are way fewer episodes of Eddington-limited accretion in $A G N \_h i g h F B$ than in $A G N \_$fid. Hence, we find that SMBHs on the local $M_{\mathrm{BH}}-M_{\star}$ relation have accretion rates which are lower than those characterising high-z quasars (e.g. Mazzucchelli et al. 2017; Vito et al. 2019, see also Table B1).

\subsubsection{The $M_{\mathrm{BH}}-M_{\star}$ relation}

Figure 11 shows the $M_{\mathrm{BH}}-M_{\star}$ relation. We analyse results for the reference simulation $A G N_{-}$fid (red points, on the left) and for the model $A G N \_h i g h F B$ (green, on the right), at redshift $z=6$. Each cirle pinpoints a $\mathrm{BH}$ in the simulation as a function of the stellar mass of the subhalo in which the $\mathrm{BH}$ resides (as provided by the SUBFIND algorithm). We consider only those BHs whose distance from the centre of their host subhalo is smaller than twice the half-mass radius of the subhalo itself (not to include BHs wandering because of spurious, numerical effects; see Section 2.2.2). Assuming a linear relation of $\log \left(M_{\mathrm{BH}}\right)$ with $\log \left(M_{*}\right)$, we find the following best-fit parameters (considering BHs with $M_{\mathrm{BH}}>$ $\left.5 \cdot 10^{5} \mathrm{M}_{\odot}\right): \log \left(M_{\mathrm{BH}}\right)=1.656 \log \left(M_{*}\right)-8.615$ for $A G N_{-}$fid, and $\log \left(M_{\mathrm{BH}}\right)=0.806 \log \left(M_{*}\right)-1.237$ for $A G N \_$highFB .

The mass of the central, most massive $\mathrm{BH}$ in our models and the stellar mass of its host subhalo are listed in Table 3. The lowest mass BHs in Figure 11 are BHs whose mass corresponds to the seed value (see Section 2.2.2). As discussed in Sections 2.2.2 and 2.3, we calibrated the feedback efficiency of the AGN model in our fiducial run so that the final mass of the most massive BH was large enough to meet the $M_{\mathrm{BH}}-M_{\star}$ relation observed at high-redshift.

We compare predictions from our simulations to observations at $z=6$ and in the local universe. As for high-redshift observations, we show best-fit relations found by Wang et al. (2010) and by Pensabene et al. (2020). The normalization of the $M_{\mathrm{BH}}-M_{\star}$ relation in the low-redshift universe is lower than that inferred from observations at $z=6$ by a factor of $\sim 15$ (Wang et al. 2010, see also Section 4).

At high redshift, the $M_{\mathrm{BH}}-M_{\star}$ relation in our simulations is shaped entirely by quasar feedback, which controls the $\mathrm{BH}$ growth while leaving SF almost unaffected (due to its inability to hamper the cosmological infall, see Section 3.4). The slope of the $M_{\mathrm{BH}}-M_{\star}$ relation inferred from our reference simulation $A G N_{-}$fid is steeper than that suggested by observations in the local universe (and usually assumed when inferring the normalization of this relation with highz data, e.g. Pensabene et al. 2020). This implies that lower mass $\mathrm{BHs}$ experience a mass growth which is not as fast as suggested by observations at low redshift. We note that BHs in the model $A G N \_h i g h F B$ at $z>6$ do not shape a $M_{\mathrm{BH}}-M_{\star}$ relation with a slope considerably steeper than that at $z=6$, nor comparable with that characterising the model $A G N_{-} f i d$ at $z=6$. This suggests that it is unlikely that $\mathrm{BHs}$ lying on a $M_{\mathrm{BH}}-M_{\star}$ relation whose slope is in agreement with that suggested by observations in the local universe have undergone a stage in which $\mathrm{BHs}$ of different mass were growing at a different pace.

Physical processes occurring on scales relevant for the $\mathrm{BH}$ accretion process may be responsible for the aforementioned trend we find in the $A G N_{-}$fid model. On the other hand, processes not included in our simulations may represent a caveat for our findings. For instance, if the quasar radiative efficiency $\epsilon_{\mathrm{r}}$ depended on the $\mathrm{BH}$ spin and thus increased with the BH mass (e.g. Davis \& Laor 2011), lower mass BHs would have a smaller efficiency and grow more because of less quasar feedback. Upcoming data are needed and crucial to confirm the possible deviation from the slope of the local $M_{\mathrm{BH}}-M_{\star}$ relation suggested by our fiducial model.

\subsection{Inflow and outflow}

In this Section, we analyse properties of inflowing and outflowing gas in four simulations introduced in Section 3.1.

Figure 12 shows the distribution of radial velocities of gas 

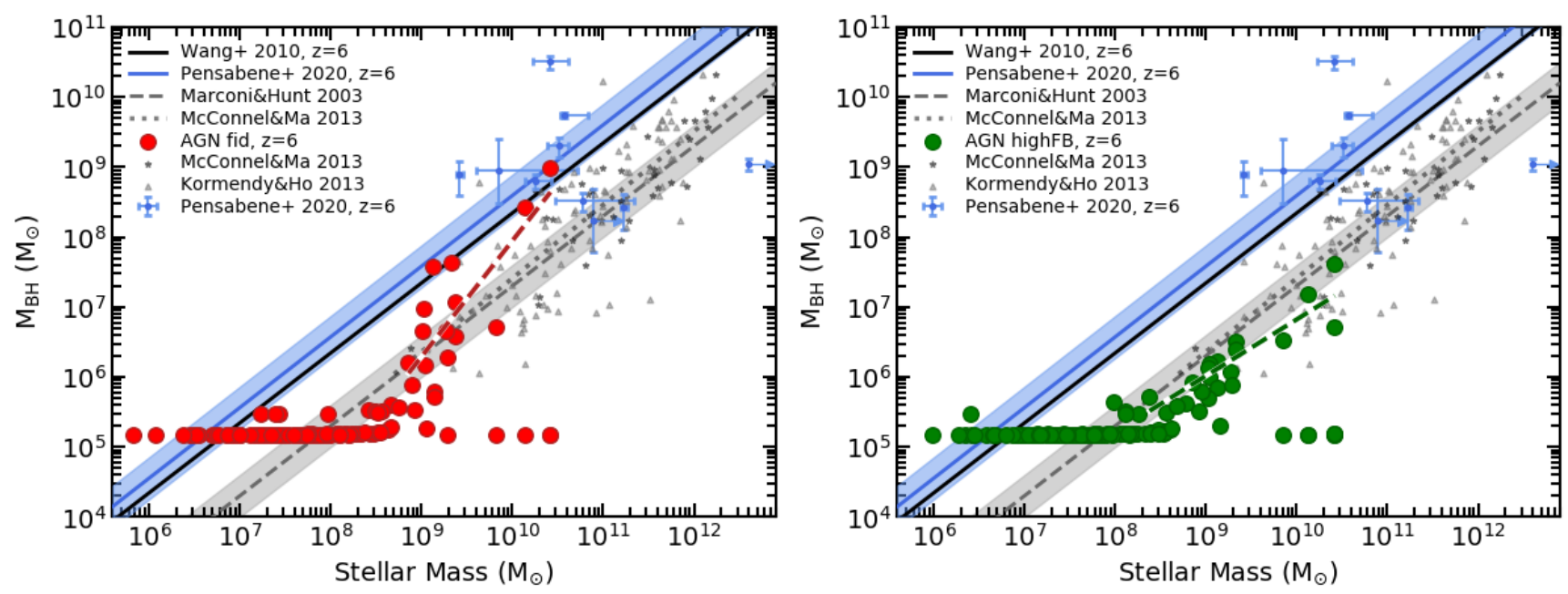

Figure 11. $M_{\mathrm{BH}}-M_{\star}$ relation for the reference simulation $A G N_{-}$fid (left) and for the model $A G N \_h i g h F B($ right), at redshift $z=6$. Red and green circles are results from the two simulations, the dashed line (same colour) showing the best fit for each model. We also show best-fit relations from high-redshift observations by Wang et al. (2010) (solid black line) and by Pensabene et al. (2020) (light-blue line, with the shaded region highlighting the $1 \sigma$ uncertainty and the light-blue symbols representing the sample used to infer the best fit). Dashed and dotted grey lines show the local $M_{\mathrm{BH}}-M_{\star}$ relations inferred by Marconi \& Hunt (2003) (the shaded envelope being the scatter around it) and by McConnell \& Ma (2013) (using a sample of early-type galaxies, pinpointed by grey stars). Grey triangles are observations by Kormendy \& Ho (2013) for low-redshift ellipticals and late-type galaxies.

as a function of the distance from the centre of the main subhalo for the different models, at $z=6$. We consider gas within the virial radius (upper panels) and within $0.1 \mathrm{r}_{\mathrm{vir}}$ (lower panels). We show that gas which is outflowing (i.e. which has a positive radial velocity $v_{\text {rad }}$ ) can reach velocities as high as $\sim 1500 \mathrm{~km} / \mathrm{s}$ should the quasar feedback be included (model $A G N_{-} f i d$ ), while velocities are on average lower when only the stellar feedback is accounted for (models $S F \_o n l y$ and $S F \_o n l y \_l o w F B$ - see also below). As for the simulation $B H s \_n o F B$, the enhanced (compared to e.g. $S F \_o n l y$ ) velocities of outflowing gas are due to the central SMBH which increases the gravitational potential and heats the gas up to higher temperatures (see Figure 3). In addition, the ISM in this latter model has not been enriched in heavy elements as in other models (due to a lower SFR, see Figure 4), and thus it is easier for stellar feedback to accelerate it up to larger speeds.

We distinguish between single-phase and multiphase outflowing/inflowing gas. Single-phase gas within $r_{v i r}$ has a temperature ranging between $10^{6}-10^{8} \mathrm{~K}$ (see for instance Figure 5, middle left panel), while the bulk of multiphase gas has a temperature $\leq 10^{5} \mathrm{~K}$; in particular, cold $\left(T_{\mathrm{c}}=300 \mathrm{~K}\right.$, see Section 2.2.1) and molecular gas represent almost $\sim 90 \%$ of the mass budget of multiphase particles in our model (see Figure 7), so it is possible to identify cold gas in Figure 12 with gas whose temperature is of few hundreds K. The figure illustrates that different phases have different kinematics: the hot and diffuse gas has higher velocities, which can easily exceed the escape velocity of the halo; multiphase gas is characterised by lower velocities, only in a few cases exceeding $\sim 300 \mathrm{~km} / \mathrm{s}$, and makes up for the almost totality of the inflowing gas. Escape velocities for the different models are listed in Table 5. We stress that the velocity of gas in our model, in good agreement with observations (see below), is the result of the modelling of feedback processes and of the advanced treatment of SPH included in our simulations. Indeed, within our feedback prescriptions, we do not assume any ad hoc wind velocity, nor we kick particles to a defined velocity suggested by observations or theoretical models (see Murante et al. 2015; Valentini et al. 2017, 2020, for further details).
Table 5. Outflowing gas mass fractions for different simulations (Column 1) at $z=6$. Column 2: escape velocity $v_{\mathrm{esc}}$ of the main subhalo. Columns 3 and 4: fraction of outflowing gas mass with radial velocity exceeding $v_{\mathrm{esc}}$, within $\mathrm{r}_{\mathrm{vir}}$ and $0.1 \mathrm{r}_{\mathrm{vir}}$, respectively. Columns 5 and 6 : fraction of outflowing gas mass with radial velocity exceeding $1000 \mathrm{~km} / \mathrm{s}$, within $r_{v i r}$ and $0.1 \mathrm{r}_{\mathrm{vir}}$, respectively.

\begin{tabular}{|c|c|c|c|c|c|}
\hline \multirow[t]{2}{*}{ Simulation } & \multirow{2}{*}{$\begin{array}{c}\mathrm{v}_{\mathrm{esc}} \\
(\mathrm{km} / \mathrm{s})\end{array}$} & \multicolumn{2}{|c|}{$\frac{\mathrm{M}_{\mathrm{gas}}\left(\mathrm{v}_{\mathrm{rad}}>\mathrm{v}_{\mathrm{esc}}\right)}{\mathrm{M}_{\mathrm{gas}}\left(\mathrm{v}_{\mathrm{rad}}>0\right)}$} & \multicolumn{2}{|c|}{$\frac{\mathrm{M}_{\mathrm{gas}}\left(\mathrm{v}_{\mathrm{rad}}>1000 \mathrm{~km} / \mathrm{s}\right)}{\mathrm{M}_{\mathrm{gas}}\left(\mathrm{v}_{\mathrm{rad}}>0\right)}$} \\
\hline & & $\begin{array}{l}r<r_{\text {vir }} \\
\left(10^{-2}\right)\end{array}$ & $\begin{array}{c}\mathrm{r}<0.1 \mathrm{r}_{\mathrm{vir}} \\
\quad\left(10^{-2}\right)\end{array}$ & $\begin{array}{l}r<r_{\text {vir }} \\
\left(10^{-3}\right)\end{array}$ & $\begin{array}{c}\mathrm{r}<0.1 \mathrm{r}_{\mathrm{vir}} \\
\quad\left(10^{-3}\right)\end{array}$ \\
\hline$B H s \_n o F B$ & 421.3 & 2.53 & 3.01 & 0.65 & 1.51 \\
\hline$A G N_{-} f i d$ & 423.4 & 3.79 & 3.16 & 1.58 & 1.7 \\
\hline SF_only & 418.4 & 3.78 & 2.24 & 0.25 & 0.2 \\
\hline$S F$ only low $F B$ & 418.8 & 1.78 & 1.44 & 0.0 & 0.0 \\
\hline
\end{tabular}

Figure 13 shows the histograms of the radial velocities of gas within different regions around the main halo of the four simulations $B H s \_n o F B$ (blue), $A G N_{-}$fid (red), $S F \_o n l y$ (light blue), and $S F \_o n l y \_l o w F B$ (orange), at $z=6$. We analyse inflowing and outflowing gas in terms of both gas mass fraction (top panels) and metal mass fraction (bottom panels). We consider the velocity distribution for all the gas in the computational volume (left panels), for the gas within the virial radius (middle panels), and for the gas within $0.1 r_{\text {vir }}$ (right panels). We find that including quasar feedback results in gas outflowing at higher velocities within all the considered volumes, with respect to models that only account for stellar feedback.

Table 5 lists the outflowing gas mass fractions for different simulations. We quantify the mass of gas which is outflowing with radial velocity exceeding either the escape velocity of the halo or a reference threshold velocity of $1000 \mathrm{~km} / \mathrm{s}$ (over the total outflowing gas), considering gas within the virial radius and $0.1 \mathrm{r}_{\mathrm{vir}}$. The table summarises how remarkable the role of AGN feedback in driving 


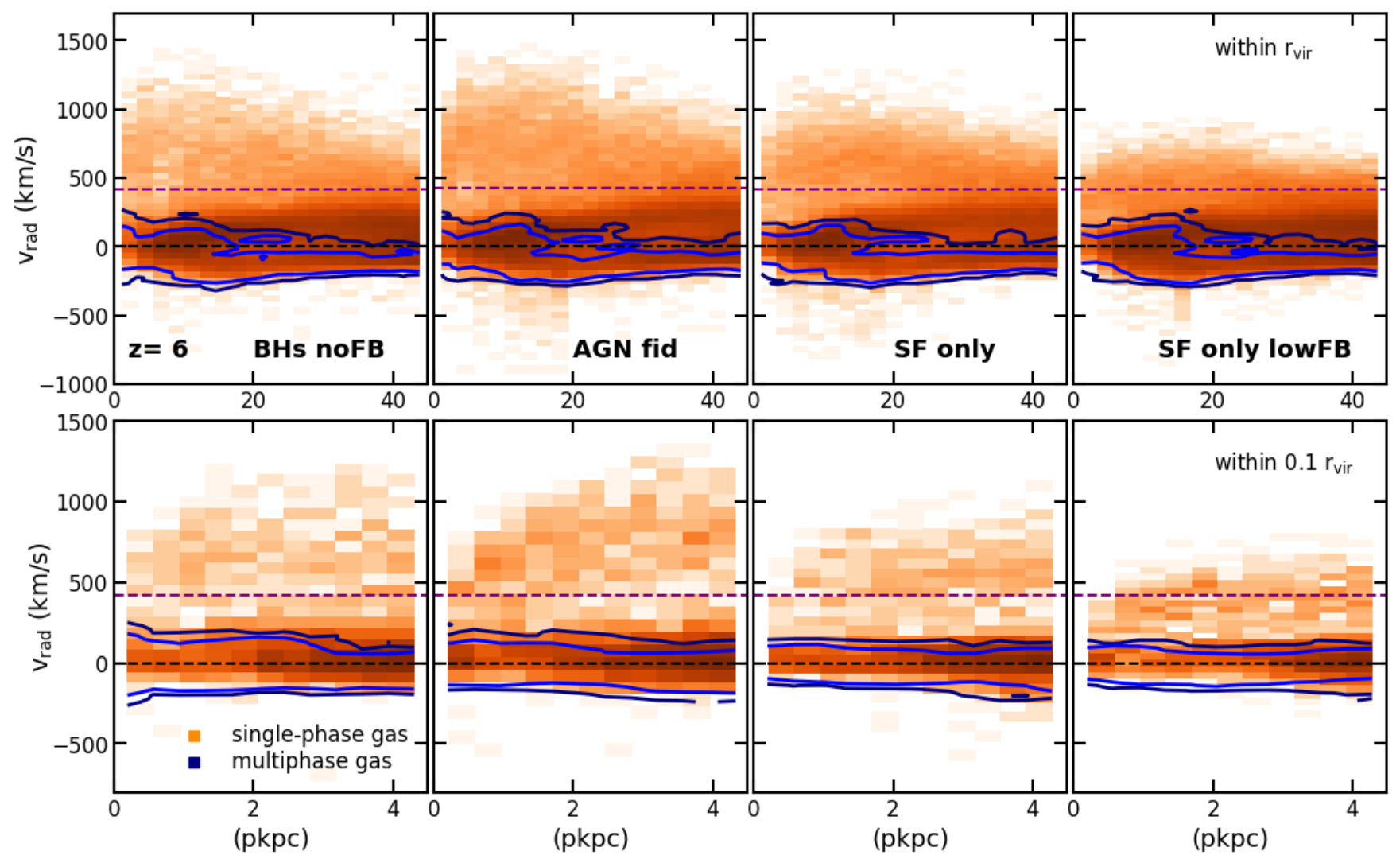

Figure 12. Radial velocity for single-phase gas particles (i.e. hot gas) and multiphase particles (i.e. cold gas, see the text for details) as a function of the distance from the centre of the main halo, at $z=6$. The background histogram shows the distribution of single-phase gas, the colour encoding the fraction of particles in each bin with respect to the total number of single-phase particles (darker shades pinpoint bins with a larger number of particles). Blue contours overlapping the background histogram show the distribution of multi-phase gas. From left to right we consider models: $B H s_{-} n o F B, A G N_{-} f i d, S F \_o n l y$, and $S F \_$only_lowFB. We consider gas within the virial radius (top panels), and within $0.1 \mathrm{r}_{\mathrm{vir}}$ (bottom panels). The horizontal, dashed black line marks $v_{\mathrm{rad}}=0 \mathrm{~km} / \mathrm{s}$, thus separating inflow from outflow. For each model, the horizontal, dashed purple line highlights the escape velocity of the halo. Escape velocities range from 418.4 to $423.4 \mathrm{~km} / \mathrm{s}$, according to the model.

outflowing gas to higher velocity is. We also find a larger outflowing gas mass fraction when the kinetic stellar feedback imparts higher velocities to gas surrounding SF sites (model $S F \_$only wrt $\left.S F \_o n l y \_l o w F B\right)$. However, assuming a stronger or weaker stellar feedback does not impact on outflowing gas velocity as significantly as the inclusion of quasar feedback does, especially when velocities above $1000 \mathrm{~km} / \mathrm{s}$ are considered.

As a caveat, we note that fractions of outflowing gas mass with radial velocity larger than the local $v_{\text {esc }}$ listed in Table 5 (columns 3 and 4) actually provide upper limits. Indeed, it cannot be excluded that gas initially moving at $v_{\text {rad }}>v_{\text {esc }}$ can eventually be slowed by ambient gas entrainment. We also note that velocity thresholds used to investigate outflow properties are relevant for final results. While we adopt $v_{\text {rad }}>0$ to distinguish between outflowing and inflowing gas, we acknowledge the possibility that a fraction of the gas orbiting in a deep potential well (as the one of our host systems) can reach $v_{\text {rad }}<200 \mathrm{~km} / \mathrm{s}$ due to gravitational motions.

As for gas inflow, we do not observe a significant difference between models $A G N_{-}$fid and $S F_{-}$only. Indeed, we find that while being responsible for driving a larger amount of gas to outflow with larger speed, quasar feedback does not impact on inflowing $\left(v_{\text {rad }}<0\right)$ gas. As different models have almost the same amount of (mainly cold) gas inflowing into the forming galaxy, they are experiencing a comparable accretion of gas from the large-scale environment. Hence, at $z=6$, quasar feedback is not yet capable of hampering the cosmological infall. This is also the main reason why we observe a similar SFH for the two aforementioned models, the amount of gas which fuels SF being still comparable down to $z=6$. We expect that gas infall from the large-scale structure will be halted at lower redshift, and that this will contribute to suppress the SF along with the additional activity of quasar feedback, whose longterm effect will be crucial at hindering gas accretion from outside and heating up the gas inside the galaxy (see also Section 4).

\section{DISCUSSION}

\subsection{The role of quasar and stellar feedback}

The AGN activity resulting from our simulations can produce episodes of both negative and positive feedback: this finding is worth to be highlighted. Despite the negligible impact that AGN feedback has on the SFH of the host galaxy down to $z=6$, we find that the SF within $0.1 \mathrm{r}_{\mathrm{vir}}$ in the reference model $A G N_{-}$fid not only can be suppressed, but also enhanced with respect to the simulation $S F \_$only (Figure 4). Quasar feedback energy can suppress temporarily the SF because it heats up the gas (see e.g. Figures 6 and B3). However, it can also enhance the SFR because it overpressurizes 

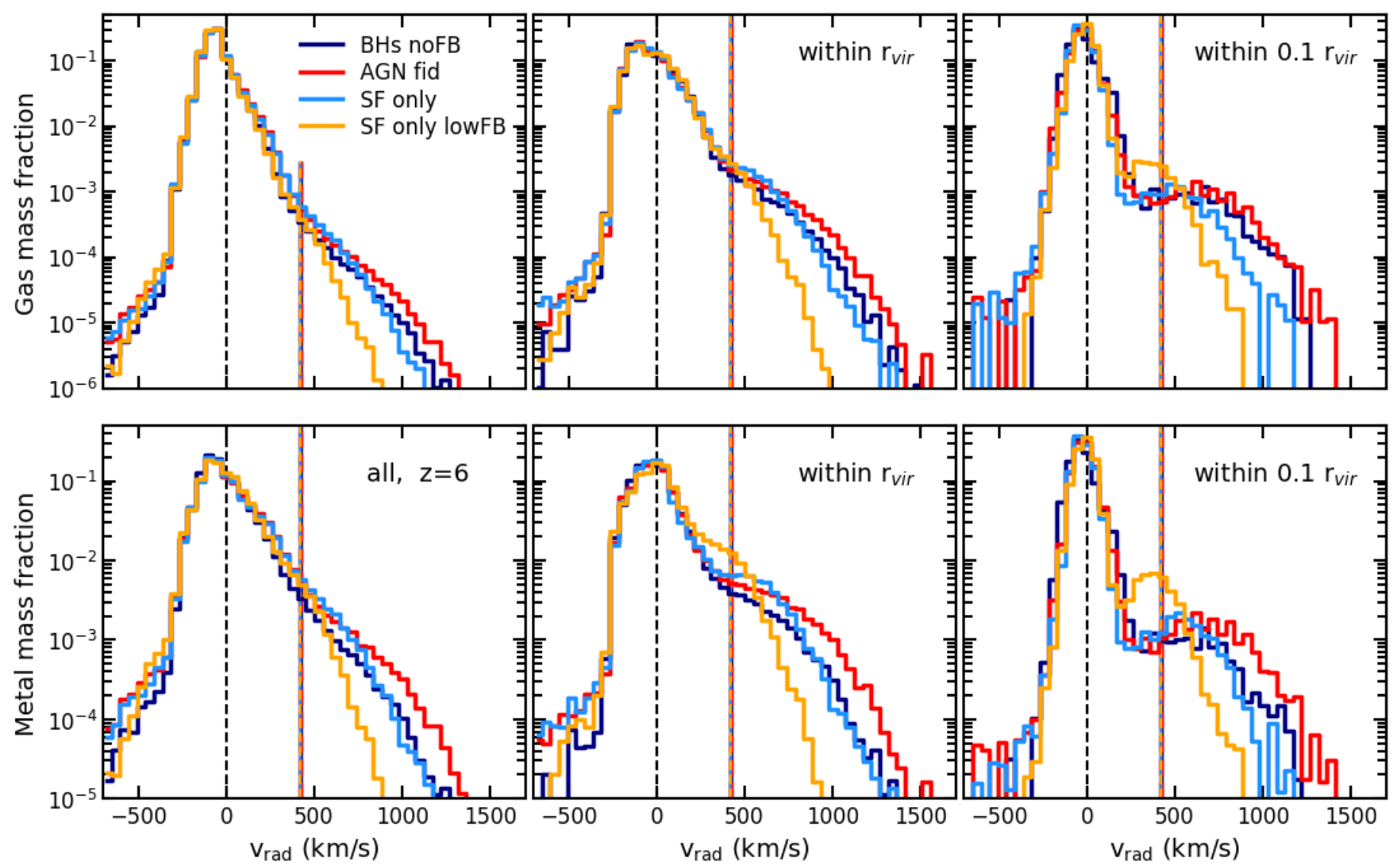

Figure 13. Histogram of the radial velocity of gas (in terms of gas mass fraction - top panels, and metal mass fraction - bottom panels) within different regions around the main halo, at $z=6$. We consider the four models $B H s \_n o F B$ (blue), $A G N \_f i d$ (red), $S F \_o n l y$ (light blue), and $S F \_o n l y \_l o w F B$ (orange). We analyse: all the gas particles in the volume (left panels), gas within the virial radius (middle panels), and gas within $0.1 \mathrm{r}_{\mathrm{vir}}$ (right panels). The vertical, dashed black line marks $v_{\mathrm{rad}}=0 \mathrm{~km} / \mathrm{s}$, hence distinguishing between inflow and outflow. For each model, the vertical line highlights the escape velocity of the halo (colours as in the legend). Escape velocities range between 418.4 and $423.4 \mathrm{~km} / \mathrm{s}$, according to the model.

the star-forming gas ${ }^{10}$. This result is also in line with Bischetti et al. (2020), where an increased SF efficiency with respect to main sequence galaxies is observed in a sample of hyper-luminous quasars $(4>z>2)$.

Considering the small impact that quasar feedback has on final properties of its host galaxy (at $z=6$, on spatial scales of several pkpc), it can be interesting to investigate whether this result stems from the choice of the quasar feedback (and/or radiative) efficiency in our modelling. As already discussed in Section 3.3, we adopted efficiency values to match $\mathrm{BH}$ masses on the $M_{\mathrm{BH}}-M_{\star}$ relation observed at high-redshift by Wang et al. (2010); Pensabene et al. (2020). To quantify the relative importance of the SN and AGN feedback processes, we proceed as follows. The typical energy input per unit time injected by $\mathrm{SN}$ explosions within the virial radius in the model $A G N_{-}$fid at $z=6$ reads:

$\dot{E}_{*}=\left(f_{\mathrm{fb}, \text { therm }}+f_{\mathrm{fb}, \mathrm{kin}}\right) E_{\mathrm{SN}} \mathrm{SFR} / M_{\star, \mathrm{SN}} \simeq 1.77 \cdot 10^{43} \mathrm{erg} / \mathrm{s}$,

where approximate values of SFR $=200 \mathrm{M}_{\odot} / \mathrm{yr}$ and $M_{\star, \mathrm{SN}}=$ $120 \mathrm{M}_{\odot}$ have been assumed (see Section 2.2.1 for further details). In the same simulation, AGN feedback supplies energy at the following rate:

$\dot{E}_{\mathrm{AGN}}=\epsilon_{\mathrm{f}} \epsilon_{\mathrm{r}} \dot{M}_{\mathrm{BH}} c^{2} \simeq 0.61 \cdot 10^{43} \mathrm{erg} / \mathrm{s}$.

10 See Valentini et al. (2020) for details, and for other similar evidence in previous numerical works.
Since $\dot{E}_{\mathrm{AGN}} / \dot{E}_{*} \simeq 0.35$, this explains why the effect of the quasar feedback is subdominant with respect to that of $\mathrm{SNe}$ at $z=6$. The relative contribution between AGN and stellar feedback increases when considering spatial scales smaller than $r_{v i r}$. In fact, in this case, $\dot{E}_{*}$ decreases as the SFR is lower (see Figure 4) while $\dot{E}_{\mathrm{AGN}}$ remains unchanged.

The aforementioned estimate can be evaluated for all the other simulations that we carried out, by exploiting results listed in Table B1. The impact of changing the quasar feedback efficiency can be appreciated for instance by comparing simulations $A G N \_$fid and $A G N \_h i g h F B$. We find that when a larger (by a factor of 10) $\epsilon_{\mathrm{f}}$ is adopted, the impact of AGN feedback on the properties of the galaxy host (e.g. the distribution of gas particles in the density-temperature plane) is not significantly different with respect to the reference $A G N_{-}$fid. This can be explained by considering that $\dot{E}_{\mathrm{AGN}}$ depends linearly on both $\epsilon_{\mathrm{f}}$ and $\dot{M}_{\mathrm{BH}}$ (i.e. $\dot{E}_{\mathrm{AGN}} \propto \epsilon_{\mathrm{f}}$, while $\dot{E}_{\mathrm{AGN}} \propto M_{\mathrm{BH}}^{2}$ ), and that the $\dot{M}_{\mathrm{BH}}$ of the most massive $\mathrm{BHs}$ in the two simulations differ by a factor $\sim 400$ (see Table B1). In conclusion, the result that quasar feedback does not affect significantly the final properties of the host galaxy does not depend on our choice of AGN feedback efficiencies, tuned to reproduce the $M_{\mathrm{BH}}-M_{\star}$ relation observed at high-redshift. Rather, our findings suggest that setting the SMBH on the observed $M_{\mathrm{BH}}-M_{\star}$ relation implies that its feedback is subdominant with respect to stellar feedback.

The lack of SF quenching in the simulation $A G N_{-}$fid with respect to $S F \_$only does not exclude that $\mathrm{SF}$ can be shut down at 
$z<6$. A more cumulative and long-term impact of AGN feedback on the host galaxy can later suppress SF.

The way in which AGN feedback is numerically implemented in our code contributes to determine the results discussed so far. Kinetic energy deposition, not included in this work, might be an important addition to the thermal one considered here. Since the kinetic injection of AGN feedback energy is expected to produce stronger signatures (kinetic energy thermalising by construction later and at larger scales; see Section 1), we predict that simulations adopting only a mechanical AGN feedback have a significantly higher impact on the host galaxy. We envisage that in a hybrid scenario where thermal and mechanical AGN feedback act in tandem to shape BH and galaxy evolution, the kinetic feedback is crucial to eject gas from the innermost regions of forming structures, thus reducing the surrounding gas column density and contributing to quench SF. We note that it is not straightforward to numerically achieve the joint activity of thermal and kinetic AGN feedback in cosmological simulations: for instance, accurate hybrid models (e.g. Weinberger et al. 2017) as the one adopted in the Illustris-TNG simulations (Pillepich et al. 2018a) which consider the $\mathrm{BH}$ accretion rate to discriminate whether the feedback has to be thermal or kinetic, would result in a thermal AGN feedback only with accretion rates characterising quasars (see e.g. Figure 10). Another possible direction of investigation and improvement is represented by the way in which AGN feedback energy is provided to the gas surrounding the BH. In fact, as the resolution of simulations increases, the resolution elements around the BH which are provided with AGN feedback energy occupy an always smaller region. The reduced volume where feedback energy is injected can play a role in determing to what extent the AGN feedback is effective, and the farthest scale affected by the process. The investigation of these effects is postponed to a forthcoming work. We also postpone to an upcoming study a detailed analysis of inflow and outflow rates, with the final goal of comparing predictions from our simulations to available estimates from observations.

As for the expected number density of UV low-luminosity quasars, the intrinsic (dust unabsorbed) UV magnitude of the most massive $\mathrm{BH}$ in the simulation $A G N_{-} f i d$ is $M_{\mathrm{UV}}=-25.6$ at $z=6$ (Section 3.3.1); we expect a corresponding observed (dust extinguished) UV magnitude $M_{\mathrm{UV} \text {,obs }} \simeq-24$, at $z=6$ (Di Mascia et al. 2021b). Quasars of this magnitude correspond to the low-luminosity tail explored by Matsuoka et al. (2016), and to a number density of $\sim 10^{-8} \mathrm{Mpc}^{-3}$ at $z=6$. The latter number density exceeds the number density of haloes with $\sim 10^{12} \mathrm{M}_{\odot}$ at $z=6$ (as in our suite of simulations) by a factor of $\sim 10^{2}$ (e.g. Angulo et al. 2012).

This result does not imply that our SMBH growth and feedback model overestimates the number density of $z \sim 6$ quasars, since the aforementioned numbers can be reconciled either assuming that the duty-cycle $\left(\tau_{D C}\right)$ of SMBHs hosted in $\sim 10^{12} \mathrm{M}_{\odot}$ haloes is short $\left(\sim 10^{-2}\right)$ at $z=6$, or that our $A G N_{-}$fid run can be considered representative only of one out of $\sim 100$ of them.

Figure 10 shows that $\tau_{D C} \sim 1$ for $A G N_{-} f i d$, for values of the Eddington-ratio $\left(\lambda_{\text {Edd }}=\dot{M}_{\mathrm{BH}} / \dot{M}_{\text {Edd }} \sim 0.01-0.1\right)$ typically adopted to distinguish between on/off AGN activity (e.g. Delvecchio et al. 2020) or high-/low-accretion phases (see Section 10). As a consequence, the first hypothesis is unlikely, unless $\lambda_{\text {Edd }} \simeq 1$ is considered as the threshold to asses whether the quasar is active.

The second hypotesis is instead supported by the results reported in Table B1: haloes with $\sim 10^{12} \mathrm{M}_{\odot}$ at $z=6$ do not necessarily always host quasars as luminous as that in our $A G N_{-}$fid model. Our simulation $A G N_{-}$fid has been designed to investigate the evolution of a BH which grows supermassive (to $\sim 10^{9} \mathrm{M}_{\odot}$ ) by $z=6$ in a $\sim 10^{12} \mathrm{M}_{\odot}$ halo. To this goal, $\mathrm{BH}$ radiative and feedback efficiencies have been tuned to the adopted values (Sections 2.2.2, 2.3 and 3.3.3).

\subsection{Comparison with observations}

Several observations suggest the presence of SFR- and/or AGNdriven outflows within the ISM of galaxies and AGN, at low and high redshift. However, no striking differences have been so far outlined between different systems, also because of the loosely constraining, available data. Here, we revise the most recent observational results in normal star-forming galaxies and AGN, and compare them with predictions from our simulations.

ALMA observations of high-redshift $(5<z<6)$, normal star-forming galaxies ( $\mathrm{SFR}=10-100 \mathrm{M}_{\odot} \mathrm{yr}^{-1}$ ) show broad [CII] wings, suggestive of cold, neutral gas outflowing with velocity up to $\sim 500 \mathrm{~km} \mathrm{~s}^{-1}$ (e.g. Gallerani et al. 2018; Sugahara et al. 2019; Ginolfi et al. 2020). These results are not dissimilar from the ones inferred from local observations of SF-driven outflows, shown to correlate with the SFR, and to have typical velocity spanning the range $300-800 \mathrm{~km} \mathrm{~s}^{-1}$ in galaxies with SFR as high as $\sim 200 \mathrm{M}_{\odot} \mathrm{yr}^{-1}$ (e.g. Förster Schreiber et al. 2019). Also, Martin (2005) analyses large-scale outflows in a sample of SF-dominated ultraluminous galaxies and finds that the upper limit of the outflow velocity of the warm, neutral $\left(\mathrm{T} \leq 10^{4} \mathrm{~K}\right)$ gas is $\sim 400-500 \mathrm{~km} \mathrm{~s}^{-1}$, with quite a large scatter towards lower values. Moreover, Heckman et al. (2015) investigate far-UV absorption lines for low-redshift, starburst galaxies (with physical properties akin to those of high-redshift, Lyman Break galaxies) and infer a velocity of $\sim 350-650 \mathrm{~km} \mathrm{~s}^{-1}$ for the warm ionized phase of starburst-driven winds in galaxies having a SFR of $\sim 200 \mathrm{M}_{\odot} \mathrm{yr}^{-1}$. Finally, Cicone et al. (2016) find that the ionised gas is outflowing at $\sim 600-800 \mathrm{~km} \mathrm{~s}^{-1}$ in a large sample of normal, star-forming galaxies in the Sloan Digital Sky Survey.

Our $S F \_$only simulation predicts outflows with velocities up to $\sim 500 \mathrm{~km} \mathrm{~s}^{-1}$ driven by SFR $=200 \mathrm{M}_{\odot} \mathrm{yr}^{-1}$, consistently with the aforementioned results.

AGN observations seem to suggest that the presence of an active $\mathrm{BH}$ increases the maximum speed that galactic outflows can reach. This is in line with our result that quasar feedback is more effective than stellar feedback at driving gas to the largest outflow velocities found in our simulations $\left(v \sim 1500 \mathrm{~km} \mathrm{~s}^{-1}\right)$. Outflow velocities can be as high as $\sim 700 \mathrm{~km} \mathrm{~s}^{-1}$ in optically selected quasars at $z \sim 6$ (Stanley et al. 2019), or even more extreme ( $1000-1500 \mathrm{~km} \mathrm{~s}^{-1}$ ), as in the case of $\mathrm{J} 1148+5251$ at $z=6.4$ (Maiolino et al. 2012; Cicone et al. 2015, but see also Decarli et al. 2018; Novak et al. 2020).

At lower redshift, Fiore et al. (2017) study the connection between extended AGN winds and host galaxy properties in a sample of AGN, including hyper-luminous quasars at $2<z<3$. For systems having a SFR of $\sim 200 \mathrm{M}_{\odot} \mathrm{yr}^{-1}$ (although their SFR is not actually the instantaneous SFR as in our simulations) and a typical AGN bolometric luminosity of $10^{46}-5 \cdot 10^{47} \mathrm{erg} \mathrm{s}^{-1}$, they find that the maximum ionised wind velocity can be in the range $500-3000 \mathrm{~km} \mathrm{~s}^{-1}$. Cicone et al. (2014) investigate galactic-scale, molecular outflows in a sample of local galaxies characterised by different AGN and starburst activity and conclude that even if the AGN does not represent the dominant source of energy (see Section 4 for our models), still it can be more effective at promoting outflows than SF activity.

Overall, observations loosely constrain outflow velocity and often suggest expected velocity ranges at redshift intervals which can be different from the one we have focussed on in this work, data 
availability being larger in the local universe. The general agreement between our results and observations is remarkable, as it is the trend for outflow velocity to be higher when AGN drives winds in addition to SF activity. The comparison between predictions from simulations and observations is not straightforward for a few reasons: for instance, the spatial scale probed by observations often cannot be certainly established. Moreover, the issue of fairly comparing gas phases probed in simulations with those traced by different observables is not a trivial one. Indeed, different phases within the same resolution element are forced to move together in simulations, and thus it is not possible to take into account the case where phase coupling is not present or hot gas entrainment by the cold phase is not achieved.

\subsection{Comparison with other numerical works}

The BH accretion model that we adopt allows us to form SMBHs with masses in agreement with the observed $M_{\mathrm{BH}}-M_{\star}$ relation at $z=6$, without the need of assuming a boost factor and by even suppressing the accretion of cold gas with high angular momentum (thus improving the commonly adopted Bondi model, see Section 2.2.2). This also highlights how the AGN feeding and feedback processes are tightly linked in our simulations. In our model, the amount of quasar feedback energy coupled to the ISM which surrounds the $\mathrm{BH}$ determines directly the properties of the gas which is later accreted onto the $\mathrm{BH}$ and which are used to compute the $\mathrm{BH}$ accretion rate. Larger AGN feedback efficiencies would couple a larger amount of energy to the ambient gas: as a consequence, the gas is heated, its density decreases, and so does the $\mathrm{BH}$ accretion rate (equation (1)).

This is a key difference with respect to models that adopt a boost factor to describe the AGN feeding process (e.g. Springel et al. 2005; Di Matteo et al. 2005; Sijacki et al. 2007; Khalatyan et al. 2008; Booth \& Schaye 2009; Dubois et al. 2013; Costa et al. 2014; Barai et al. 2018; but see Lupi et al. 2019). In those models, a way larger amount of AGN feedback energy can be coupled to the gas around the $\mathrm{BH}$ without affecting directly the $\mathrm{BH}$ accretion process (whereas more evident feedback signatures on the host galaxy can be produced). Indeed, if AGN feedback heated the gas and decreased its density, the presence of a fudge factor (whose value tipically ranges from several tens to few hundreds) in those models would compensate for a low $\mathrm{BH}$ accretion rate. Since almost all the simulations adopt the $M_{\mathrm{BH}}-M_{\star}$ relation to get $\mathrm{BH}$ final masses in agreement with observations, this explains why the quasar feedback efficiency adopted in the reference model $A G N_{-}$fid is lower than commonly assumed.

Our simulation suite provides a detailed outlook on the processes of SMBH growth, quasar feedback and outflows in the early universe. Our finding that BHs can grow supermassive $\left(\sim 10^{8}-10^{9} \mathrm{M}_{\odot}\right)$ from massive $\left(\sim 10^{5}-10^{6} \mathrm{M}_{\odot}\right)$ seeds in massive $\left(\sim 10^{12} \mathrm{M}_{\odot}\right)$ DM haloes by $z=6$ via Eddington-limited gas accretion is consistent with results from several, previous simulations (e.g. Sijacki et al. 2009; Di Matteo et al. 2012, 2017; Costa et al. 2014; Smidt et al. 2018; Barai et al. 2018; Lupi et al. 2019; Zhu et al. 2020). In line with our results, works among the aforementioned ones have also shown that SMBHs often accrete at a rate which is close to the Eddington rate (e.g. Di Matteo et al. 2017; Smidt et al. 2018; Barai et al. 2018; Lupi et al. 2019), and BH$\mathrm{BH}$ mergers contribute little to the $\mathrm{BH}$ growth with respect to gas accretion (Di Matteo et al. 2012, 2017). The numerical modelling of processes driving (or hampering) the $\mathrm{BH}$ growth features differ- ences between our work and previous simulations, and among the aforementioned works themselves.

Powerful outflows in our simulations are triggered by the joint activity of stellar and quasar feedback, in agreement with results by Costa et al. (2015); Biernacki \& Teyssier (2018), who also showed that the AGN-powered component is necessary for the outflows to reach higher radial velocities. Our finding that cold gas in outflows moves with a slower speed than the hot phase component is in line with previous works; however, cold gas outflows in our simulations are slower than those predicted by Costa et al. (2015); Ni et al. (2018), both the aforementioned works retrieving for them velocities which can even exceed $\sim 1000 \mathrm{~km} / \mathrm{s}$.

As for cold gas inflow, our finding that inflowing warm and cold gas filaments feed the halo and provide the growing $\mathrm{BH}$ and the forming host galaxy with fuel is in agreement with results from previous simulations (Sijacki et al. 2009; Di Matteo et al. 2012; Dubois et al. 2013; Costa et al. 2014; Barai et al. 2018; Smidt et al. 2018). While there is general consensus on the role played by these cold streams in funnelling gas towards the innermost regions of growing structures, it is still debated whether the complex web of filaments can survive the effect of AGN feedback due to their high density (Di Matteo et al. 2012), although dynamically perturbed (Dubois et al. 2013), or can be disrupted by quasar outflows propagating in the same direction (Barai et al. 2018). Our simulations support the idea that inflowing cold gas streams cannot be halted by the joint $\mathrm{SN}$ and quasar feedback by $z=6$.

As also discussed in previous sections, quasar feedback controls the SMBH growth (see also Sijacki et al. 2009; Dubois et al. 2013) and affects to various degrees host galaxy properties (e.g. Di Matteo et al. 2012; Khandai et al. 2012; Costa et al. 2014; Curtis \& Sijacki 2016; Habouzit et al. 2019). Interestingly, quasar feedback in the aforementioned simulations is found to suppress SF little to moderatly, the full quenching being never achieved by $z \sim 6$. A longer-term AGN feedback effect is expected to be crucial to significantly suppress and even quench SF at lower redshift, as we envisage in Sections 3.4 and 4.1 (see also Curtis \& Sijacki 2016).

Although the common expectation is that the inclusion of AGN feedback results in striking differences with respect to the case where SMBH effects are not accounted for, some recent works taking advantage of state-of-the-art cosmological simulations showed that this may be not always true. Recently, Sorini et al. (2020) investigated the properties of the circumgalactic and intergalactic medium around quasars at redshift $2<z<3$ in the SIMBA simulation. Interestingly, they found that the physical properties of the gas surrounding quasars, i.e. gas density, temperature, and radial velocity out to several virial radii, are primarily shaped by stellar feedback, while the contribution from the mechanical AGN feedback (in different flavours, namely winds, jets and X-ray heating) plays a minimal role. Similar conclusions have been also drawn by Rahmati et al. (2015): when analysing the distribution of neutral hydrogen around high-redshift $(2<z<3)$ galaxies and quasars in the EAGLE simulation, they found that the neutral hydrogen covering fraction in Lyman Limit Systems is not sensitive to the effect of AGN feedback at all (out to $\sim 1 \mathrm{pMpc}$ ), while the stellar feedback is the main driver for the results. Results from Faucher-Giguère et al. (2016) are also in line with the finding that the availability of neutral hydrogen (on $100 \mathrm{kpc}$ scale) is mainly determined by the effect of stellar feedback alone: by studying properties of massive haloes $\left(10^{12}<\mathrm{M}_{\text {halo }}\left(\mathrm{M}_{\odot}\right)<10^{13}\right.$ at $\left.z=2-2.5\right)$ within the FIRE project, they found neutral hydrogen covering fractions in agreement with observations of luminous quasars and claim that a significant contribution from AGN feedback is not needed. In ad- 
dition to the aforementioned numerical studies, recent observations (e.g. Davies et al. 2020; Scholtz et al. 2020) of galaxies at $z \lesssim 2.5$ have also supported the evidence that AGN outflows can have no effect on the instantaneous SFR of the host galaxy.

\section{SUMMARY AND CONCLUSIONS}

We carried out a suite of high-resolution $\left(m_{\text {gas }}=2.89 \cdot 10^{5} \mathrm{M}_{\odot}\right.$ and $\epsilon=59 \mathrm{ppc}$ for gas particles) cosmological, zoom-in simulations of high-z galaxies $\left(M_{\text {halo, } \mathrm{DM}} \simeq 10^{12} \mathrm{M}_{\odot}\right.$, at $z=6$ ), based on the GADGET-3 code and using the MUPPI sub-resolution model to describe physical processes in a multiphase ISM, BH accretion and thermal quasar feedback. The goal of this study is to investigate the growth history of SMBHs down to $z=6$, and to quantify the impact of stellar and quasar feedback both on the quasar-host galaxy final properties and on the formation of SMBHs. Our main results can be summarised as follows:

- BHs can grow supermassive by $z=6$ and reach a final mass which is in agreement with the $M_{\mathrm{BH}}-M_{\star}$ relation observed at that redshift (Figure 11). Gas accretion is the main driver of BH growth, with mergers playing a sub-dominant role (Figure 9).

- In our reference model, $A G N_{-} f i d$, the central, most massive BH has a mass of $9.85 \cdot 10^{8} \mathrm{M}_{\odot}$, an accretion rate $\dot{M}_{\mathrm{BH}}=35.53 \mathrm{M}_{\odot} \mathrm{yr}^{-1}$ (i.e. $\dot{M}_{\mathrm{BH}} / \dot{M}_{\mathrm{Edd}}=0.495$ ), and is hosted in a galaxy whose stellar mass is $M_{*} \sim 2.6 \cdot 10^{10} \mathrm{M}_{\odot}$, at $z=6$. Such $\dot{M}_{\mathrm{BH}}$ value corresponds to an intrinsic, unextincted UV magnitude of $M_{\mathrm{UV}}=-25.6$. If the quasar feedback efficiency were tuned to produce a SMBH lying on the local $M_{\mathrm{BH}}-M_{\star}$ relation, its accretion rate at $z=6$ would fall short of the measured one in high- $z$ quasars.

- The slope of the $M_{\mathrm{BH}}-M_{\star}$ relation inferred from $A G N_{-}$fid is steeper than suggested by local observations. At high redshift, the $M_{\mathrm{BH}}-M_{\star}$ relation in our models is shaped by quasar feedback, which controls the $\mathrm{BH}$ growth while leaving SF almost unaffected (due to its inability to halt the cosmological infall).

- By comparing properties of the ISM in models with and without $\mathrm{SMBH}$, we find that the temperature of the ISM is on average higher (by a factor of $\sim 2$ within $\sim 4 \mathrm{kpc}$ from the galaxy centre) and that the total gas metallicity is lower (by a factor of 3 ) due to a reduced SFR (by $10 \mathrm{M}_{\odot} \mathrm{yr}^{-1}$ ) when AGN is not included. Properties of the host galaxy in our fiducial simulation are in good agreement with observations.

- Quasar feedback has two opposite effects on the SFH of the host galaxy: (a) by heating the gas, it quenches SF; (b) by overpressurizing the ISM, it favours the formation of new stars. However, such modulation effects are sub-dominant with respect to the rate imposed by cosmological infall (see below). As a result, feedback has only a negligible effect on the galaxy SFH. Nevertheless, quasar feedback strongly controls $\mathrm{BH}$ growth. When turned off in simulations, the final SMBH mass is found to be $\approx 100 \times$ larger.

- Galactic outflows are promoted by the joint activity of stellar and quasar feedback. We find that quasar feedback increases the outflow rate and accelerates the gas to larger velocities (Figure 13). Hot and cold phases are both involved in outflows. In addition, different phases are characterised by different kinematics (Figure 12): the hot $\left(T \gtrsim 10^{5} \mathrm{~K}\right)$ gas has velocities which can easily exceed the escape velocity of the halo and be even larger than $\sim 1000 \mathrm{~km} / \mathrm{s}$, when quasar feedback is included. On the other hand, cold and warm $\left(T \lesssim 10^{4} \mathrm{~K}\right)$ phases have lower velocities, only in a few cases exceeding $\sim 300 \mathrm{~km} / \mathrm{s}$. The imprint of quasar feedback is on the high-velocity tail of the outflowing gas distribution; this feature is present even if the AGN does not represent the dominant source of energy in the host galaxy. Predictions from our simulations as for outflow velocity are in good agreement with observations.

- Cold gas makes up for the almost totality of the infalling mass. We find that quasar feedback cannot hinder the inflow process. Models with and without SMBH activity experience a comparable accretion rate from the large-scale structure; such cosmological infall fuels SF at a comparable rate in different systems.

The suite of simulations introduced in this work will be further analysed in forthcoming papers. As new upcoming observational instruments (e.g. the James Webb Space Telescope, JWST and the European-Extremely Large Telescope, E-ELT) will allow to probe the very high-redshift Universe, it is extremely important to have simulations able to provide the theoretical counterpart and to shed light on what drives the formation and evolution of the first structures.

\section{APPENDIX A: HALOES IN THE PARENT, DM-ONLY SIMULATION}

In this Appendix, we highlight some interesting features of the parent, DM-only simulation described in Section 2.1, and discuss how we selected our target halo for the zoomed-in simulation.

Figure A1 shows the distribution of all the subhaloes in the parent, DM-only simulation identified by the SUBFIND algorithm at $z=6$. The left-hand and the right-hand panel depict the $x-y$ and $z-y$ projections, respectively. The colour of each circle encodes the subhalo mass, while its size is proportional to the virial radius of the subhalo. Distances are shown with respect to the centre of the target subhalo, located in $(x, y, z)=(0,0,0)$. As for the statistics of the subhaloes, there are 10 subhaloes more massive than $10^{12} \mathrm{M}_{\odot}$ (blue circles), 41 subhaloes whose mass exceeds $5 \cdot 10^{11} \mathrm{M}_{\odot}, 1892$ (6584) subhaloes more massive than $10^{11} \mathrm{M}_{\odot}\left(5 \cdot 10^{10} \mathrm{M}_{\odot}\right)$. The most massive subhalo in the box has a mass of $1.32 \cdot 10^{12} \mathrm{M}_{\odot}$, the target halo is as massive as $1.12 \cdot 10^{12} \mathrm{M}_{\odot}$ (ranked 8th).

When selecting the target subhalo, we excluded subhaloes with mass $>10^{12} \mathrm{M}_{\odot}$ close to the borders of the box. We also excluded massive $\left(>10^{12} \mathrm{M}_{\odot}\right.$ ) subhaloes in underdense regions, i.e. with no close, fairly-massive subhaloes, as possible candidate target subhaloes. For instance, the subhaloes ranked 2 nd, 7 th, and 9 th have been excluded because there are no subhaloes at least as massive as $8 \cdot 10^{10} \mathrm{M}_{\odot}$ within a distance of 6.5 times their own virial radius. In addition, we decided not to focus on a subhalo more massive than $10^{12} \mathrm{M}_{\odot}$ with a too-close massive companion. For instance, we excluded the most massive subhalo as possible target because $\mathrm{a} \sim 2.6 \cdot 10^{11} \mathrm{M}_{\odot}$ subhalo is located at a distance which is smaller than the sum of the virial radii of the two aforementioned subhaloes. We selected our target halo because it is massive enough to be eligible for a quasar-host galaxy, and because it has a massive $\left(\sim 7.5 \cdot 10^{11} \mathrm{M}_{\odot}\right)$ satellite subhalo $103 \mathrm{pkpc}$ far from it (this distance being larger than the sum of the virial radii of the two systems).

The proximity between massive subhaloes is indeed a very interesting topic, as several studies have suggested that extremelymassive BHs $\left(M_{\mathrm{BH}} \gtrsim 10^{8}-10^{9} \mathrm{M}_{\odot}\right)$ preferably reside in overdense regions (see e.g. Yoon et al. 2019, and references therein). Thus, we included the distance from a massive, satellite subhalo as a requirement to select the target halo. A close (non merging, see below), massive subhalo can indeed shed some light on the joint evolution of the host BHs and on their environment. This subject will be further investigated in forthcoming works, too.

To this end, Figure A2 shows the cumulative histogram of 

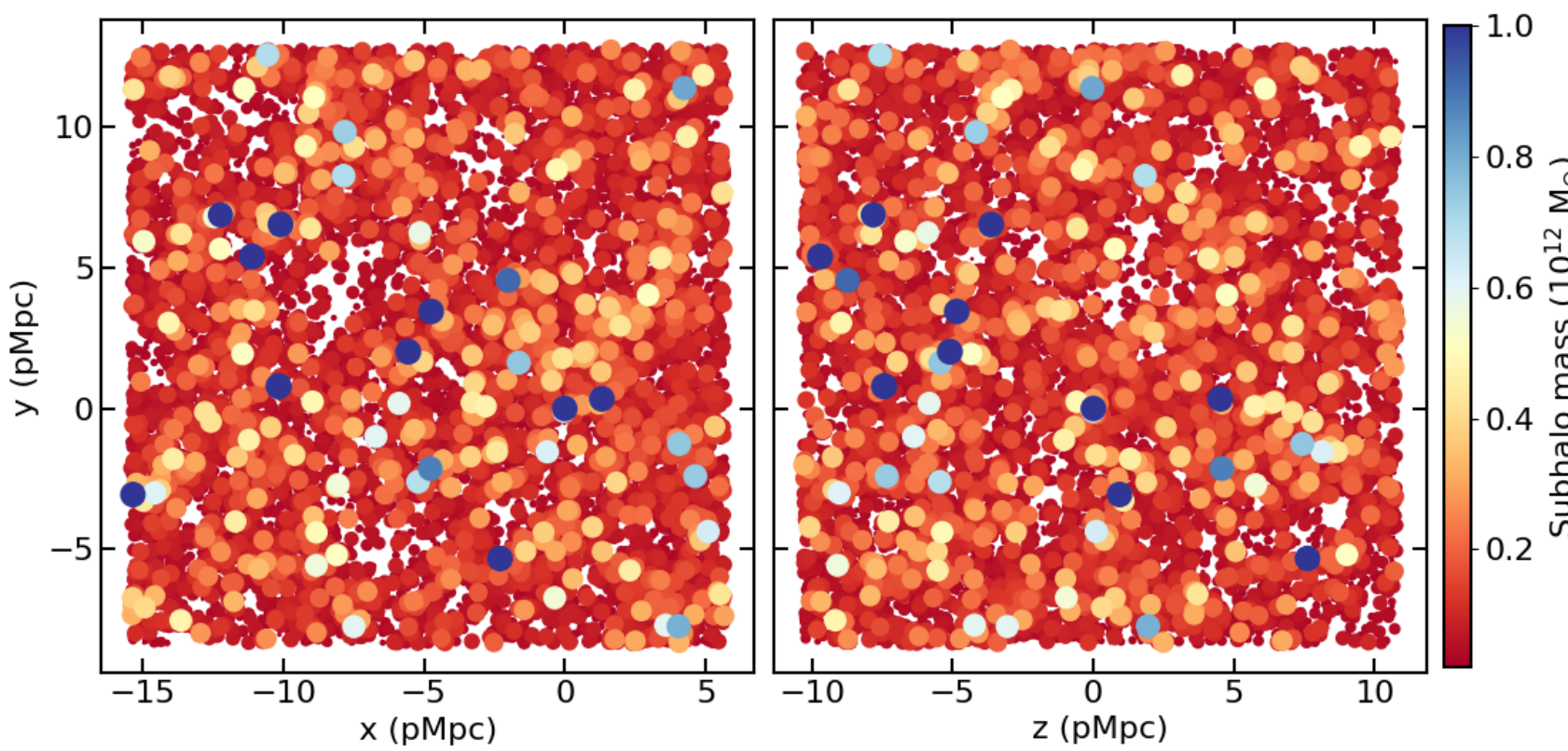

Figure A1. Projected distribution of all the subhaloes in the parent, DM-only simulation in the planes $x-y$ (left-hand panel) and $z-y$ (right-hand panel), at redshift $z=6$. The colour bar encodes the subhalo mass. The size of each circle scales with the virial radius of the subhalo. Distances are expressed in physical $\mathrm{Mpc}$ (pMpc), with respect to the centre of the subhalo that has been chosen for the zoomed simulation.

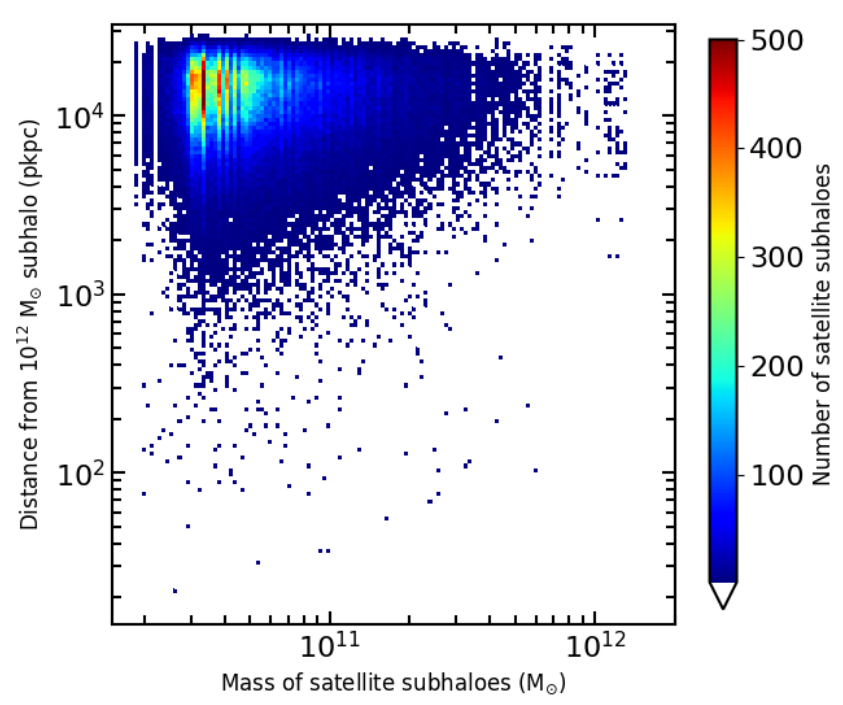

Figure A2. Cumulative 2-D histogram providing the number of satellite subhaloes per bin of mass and distance from each of the subhaloes whose mass is larger than $10^{12} \mathrm{M}_{\odot}$. This histogram has been obtained by summing up the histograms of each of the 10 subhaloes more massive than $10^{12} \mathrm{M}_{\odot}$ in the parent DM-only simulation, at $z=6$. The colour in each bin encodes the number of subhaloes.

satellite subhaloes per bin of mass and distance from each of the 10 most massive subhaloes, in the parent DM-only simulation, at $z=6$. We computed the histogram of the subhaloes surrounding each of the 10 subhaloes more massive than $10^{12} \mathrm{M}_{\odot}$ as a function of both their mass and distance, and then summed up to retrieve a more solid result. The virial radius of the 10 most massive subhaloes spans the range $46.9-50.7$ pkpc: as a consequence, if two subhaloes are closer than $\sim 10^{2}$ pkpc, they are likely interacting and will merge. Figure A2 suggests how hard it is to have close, massive subhaloes, in the volume considered by our simulation. As these close, massive subhaloes are expected to be the hosts of extremely-massive BHs, this result hints at the unlikely possibility of having extremely-massive $\mathrm{BH}$ pairs in overdense regions of the early universe. Rather, massive subhaloes (and hence extremelymassive $\mathrm{BHs}$ ) tend to reside in relatively isolated environments.

\section{APPENDIX B: OTHER SIMULATIONS}

In this Appendix we introduce Table B1, where we list the most relevant features of central galaxies and their most massive BHs for the suite of simulations that we performed to prepare this work. It also shows the parameters of the sub-resolution model that we varied (columns $2-6$ ), and how they impact on final properties of central galaxies and their BHs (columns $7-12$ ), at $z=6$.

In what follows, we focus on the simulation $S F \_o n l y$ and show some further features, to ease the comparison with the reference simulation AGN_fid discussed in Section 3.

Figure B1 shows a zoom-in on the central galaxy of the simulation $S F \_o n l y$, while Figure B2 illustrates the mass (left panels) and metallicity (right panels) distribution in the density-temperature phase diagram of gas particles in the same simulation, at redshift $z=6$. Figure B3 shows density and temperature radial profiles for the aforementioned simulation.

\section{DATA AVAILABILITY}

The data underlying this article will be shared on reasonable request to the corresponding author. 

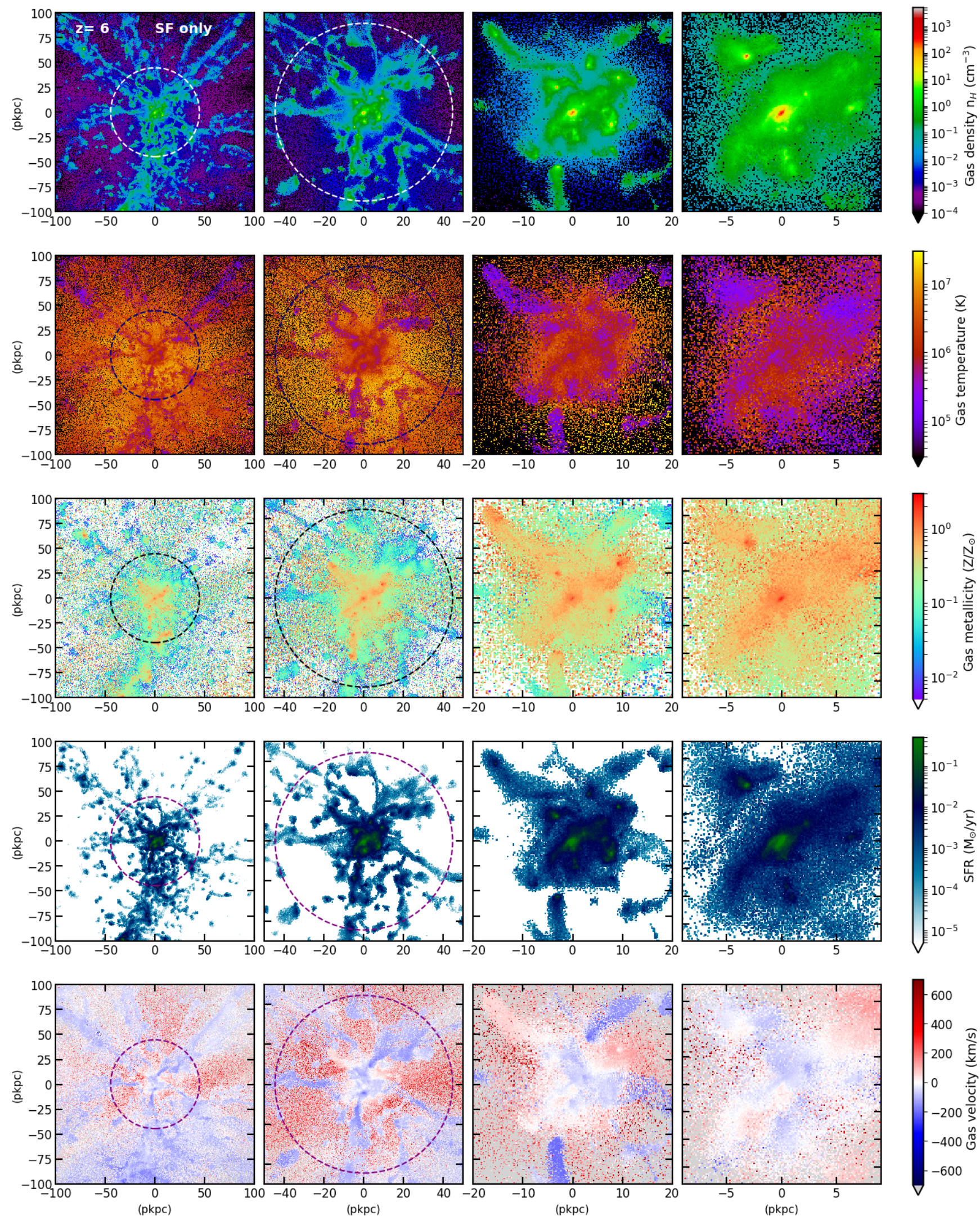

Figure B1. We show gas density (first row), gas temperature (second row), gas metallicity (third row), the SFR of gas particles (fourth row), and the massweighted, radial velocity of gas particles (bottom row) for the simulation $S F_{-}$only, at redshift $z=6$. We progressively zoom-in from left to right: the first and second columns show a box of $200 \mathrm{pkpc}$ and $100 \mathrm{pkpc}$ a side, respectively, the projection being performed along the $z$-axis (over $200 \mathrm{pkpc}$ and $100 \mathrm{pkpc}$, respectively). The dashed circumference has the virial radius of the central, target halo as a radius. The third column shows a box of $40 \mathrm{pkpc}$ (projection is over $20 \mathrm{pkpc}$ along the $z$-axis), while in the fourth column we consider a box of $18 \mathrm{pkpc}$ (projection is over $9 \mathrm{pkpc}$ along the $z$-axis). All the maps are centred on the centre of the most massive subhalo. Same as Figure 2, but for the simulation SF_only. 
Table B1. Relevant parameters adopted and main features of the simulated structures at $z=6$ in the suite of simulations carried out. Column 1: simulation. Column 2: kinetic stellar feedback efficiency. Column 3: BH radiative efficiency. Column 4: quasar feedback efficiency. Column 5: SF efficiency. Column 6: pressure of the ISM at which $f_{\mathrm{mol}}=0.5$ (see Section 2.2.1). Column 7: central BH mass. Column 8: BH accretion rate. Column 9: stellar mass within the virial radius. Column 10: SFR within $r_{\text {vir }}$. Column 11: stellar mass within $0.1 r_{\text {vir }}$. Column 12: SFR within $0.1 r_{\text {vir }}$.

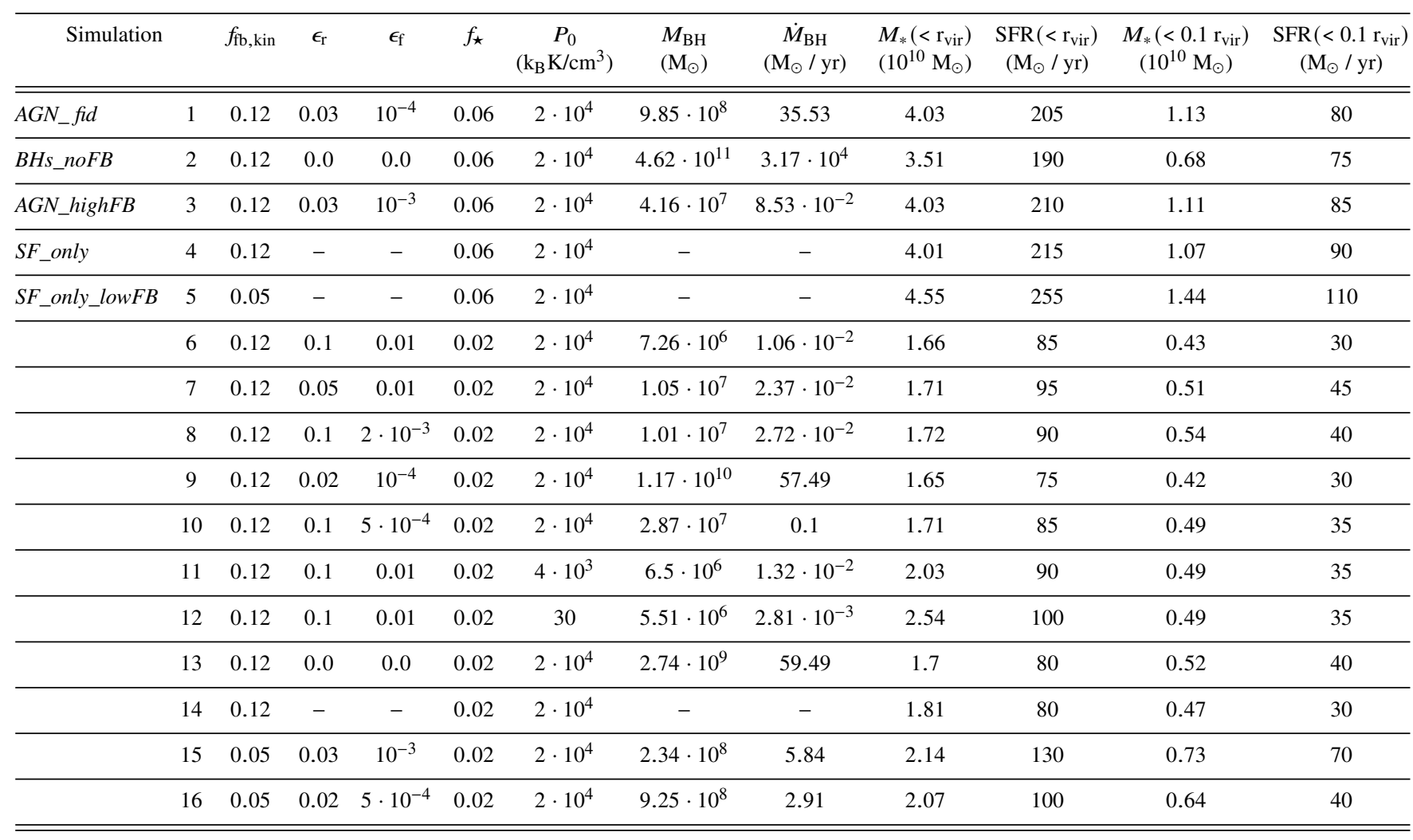

\section{ACKNOWLEDGMENTS}

We thank the anonymous referee for the prompt and careful report that helped improving the presentation of results. We are grateful to Volker Springel for making the GADGET3 code available to us. We thank Klaus Dolag, Stefano Borgani, Giuseppe Murante, and Stefano Carniani for insightful discussions. MV is supported by the Excellence Cluster ORIGINS, which is funded by the Deutsche Forschungsgemeinschaft (DFG, German Research Foundation) under Germany's Excellence Strategy - EXC-2094 - 390783311. MV also acknowledges support from the Alexander von Humboldt Stiftung and from the Carl Friedrich von Siemens Stiftung. Simulations were carried out using computational resources at Scuola Normale Superiore, and Galileo at CINECA (CPU time has been assigned through the project "Witnessing the growth of high-redshift SMBHs", PI: M. Valentini). We acknowledge the computing centre of Cineca and INAF (under the coordination of the "Accordo Quadro MoU per lo svolgimento di attività congiunta di ricerca - Nuove frontiere in Astrofisica: HPC e Data Exploration di nuova generazione"), for the availability of computing resources and support. AF acknowledges support from the ERC Advanced Grant INTERSTELLAR H2020/740120. Any dissemination of results must indicate that it reflects only the author's view and that the Commission is not responsible for any use that may be made of the information it contains. Partial support from the Carl Friedrich von SiemensForschungspreis der Alexander von Humboldt-Stiftung Research Award is kindly acknowledged.

\section{REFERENCES}

Alatalo K., et al., 2015, ApJ, 798, 31

Angulo R. E., Springel V., White S. D. M., Cole S., Jenkins A., Baugh C. M., Frenk C. S., 2012, MNRAS, 425, 2722

Bañados E., et al., 2014, AJ, 148, 14

Barai P., Murante G., Borgani S., Gaspari M., Granato G. L., Monaco P., Ragone-Figueroa C., 2016, MNRAS, 461, 1548

Barai P., Gallerani S., Pallottini A., Ferrara A., Marconi A., Cicone C., Maiolino R., Carniani S., 2018, MNRAS, 473, 4003

Barkana R., Loeb A., 2001, Phys. Rep., 349, 125

Barnett R., et al., 2015, A\&A, 575, A31

Beck A. M., et al., 2016, MNRAS, 455, 2110

Begelman M. C., Volonteri M., Rees M. J., 2006, MNRAS, 370, 289

Behroozi P. S., Wechsler R. H., Conroy C., 2013, ApJ, 770, 57

Bellovary J., Brooks A., Volonteri M., Governato F., Quinn T., Wadsley J., 2013, ApJ, 779, 136

Biernacki P., Teyssier R., 2018, MNRAS, 475, 5688

Bischetti M., Maiolino R., Carniani S., Fiore F., Piconcelli E., Fluetsch A., 2019, A\&A, 630, A59

Bischetti M., et al., 2020, arXiv e-prints, p. arXiv:2009.01112

Blitz L., Rosolowsky E., 2006, ApJ, 650, 933

Bluck A. F. L., et al., 2020, MNRAS, 499, 230

Bondi H., 1952, MNRAS, 112, 195

Bondi H., Hoyle F., 1944, MNRAS, 104, 273

Bongiorno A., et al., 2014, MNRAS, 443, 2077

Booth C. M., Schaye J., 2009, MNRAS, 398, 53

Bouwens R. J., et al., 2012, ApJ, 754, 83

Brandt W. N., et al., 2002, ApJ, 569, L5

Bromm V., Loeb A., 2003, ApJ, 596, 34

Bryan G. L., Norman M. L., 1998, ApJ, 495, 80 


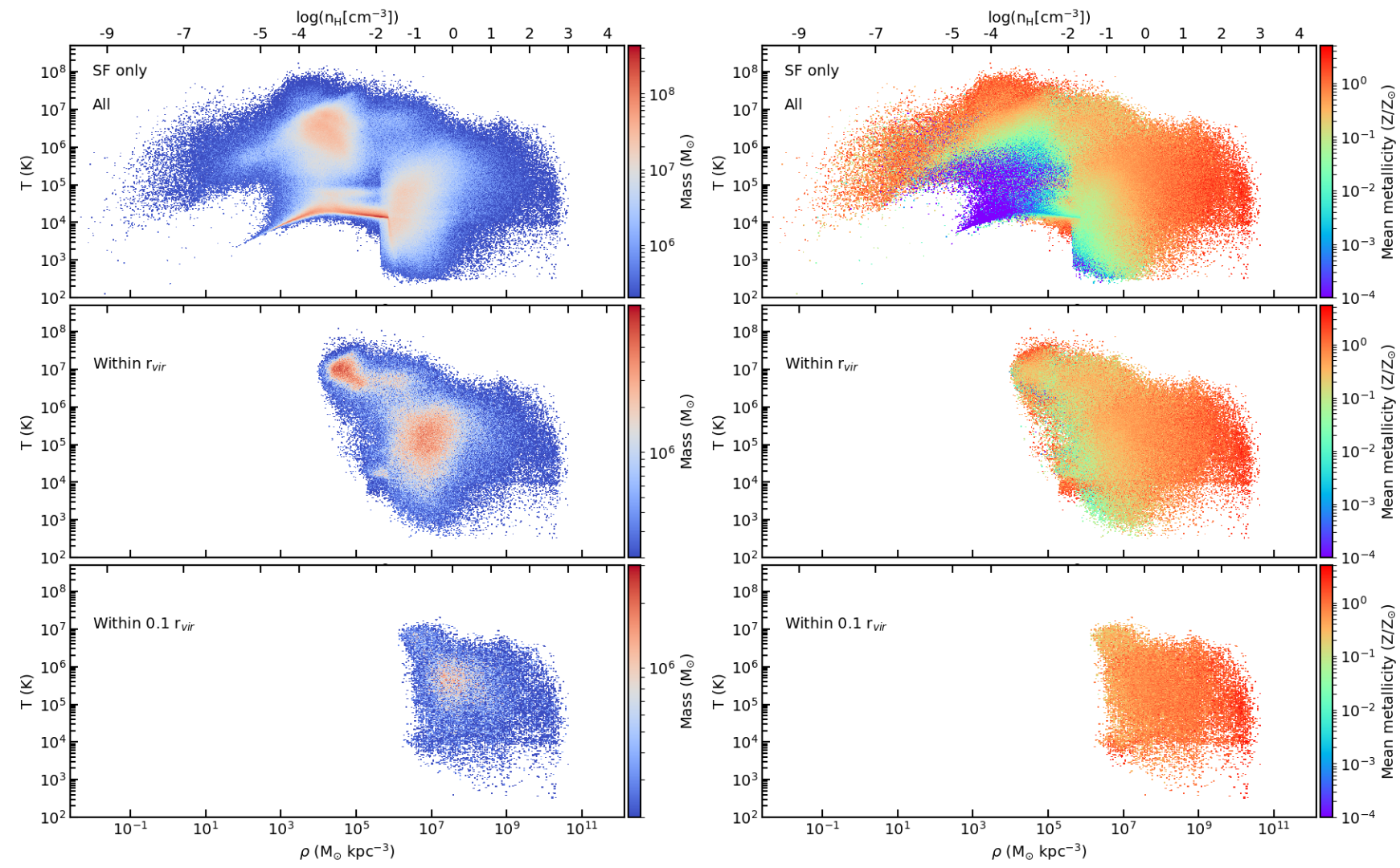

Figure B2. Distribution of gas particles in the density-temperature plane in the reference simulation $S F \_o n l y$, at redshift $z=6$. Top panels show the distribution of all the gas particles in the Lagrangian region, middle and bottom panels refer to gas particles within the virial radius $r_{v i r}$ and within $0.1 r_{v i r}$, respectively. The colour encodes the gas mass per density-temperature bin (left-hand panels) and the mean metallicity per bin (right-hand panels). All the color bars in the left set of panels share the minimum value, while the maximum of the color scale is independent for each panel, to better capture features (the same is true for the three panels on the right). Same as Figure 5, but for the simulation SF_only.

Caffau E., Ludwig H.-G., Steffen M., Freytag B., Bonifacio P., 2011, Sol. Phys., 268, 255

Carilli C. L., Walter F., 2013, ARA\&A, 51, 105

Carniani S., et al., 2019, MNRAS, 489, 3939

Chabrier G., 2003, PASP, 115, 763

Churazov E., Sazonov S., Sunyaev R., Forman W., Jones C., Böhringer H., 2005, MNRAS, 363, L91

Cicone C., et al., 2014, A\&A, 562, A21

Cicone C., et al., 2015, A\&A, 574, A14

Cicone C., Maiolino R., Marconi A., 2016, A\&A, 588, A41

Combes F., 2018, A\&ARv, 26, 5

Costa T., Sijacki D., Trenti M., Haehnelt M. G., 2014, MNRAS, 439, 2146

Costa T., Sijacki D., Haehnelt M. G., 2015, MNRAS, 448, L30

Costa T., Rosdahl J., Sijacki D., Haehnelt M. G., 2018, MNRAS, 479, 2079

Costa T., Pakmor R., Springel V., 2020, MNRAS, 497, 5229

Croton D. J., et al., 2006, MNRAS, 365, 11

Curtis M., Sijacki D., 2016, MNRAS, 457, L34

Daddi E., et al., 2010, ApJ, 714, L118

Davies R. L., et al., 2020, ApJ, 894, 28

Davis S. W., Laor A., 2011, ApJ, 728, 98

Dayal P., Ferrara A., 2018, Phys. Rep., 780, 1

De Rosa G., et al., 2014, ApJ, 790, 145

Decarli R., et al., 2018, ApJ, 854, 97

Delvecchio I., et al., 2020, ApJ, 892, 17

Di Mascia F., et al., 2021a, MNRAS, 503, 2349

Di Mascia F., et al., 2021b, MNRAS, subm.

Di Matteo T., Springel V., Hernquist L., 2005, Nature, 433, 604

Di Matteo T., Khandai N., DeGraf C., Feng Y., Croft R. A. C., Lopez J., Springel V., 2012, ApJ, 745, L29
Di Matteo T., Croft R. A. C., Feng Y., Waters D., Wilkins S., 2017, MNRAS, 467,4243

Dolag K., Borgani S., Murante G., Springel V., 2009, MNRAS, 399, 497

Dubois Y., Pichon C., Haehnelt M., Kimm T., Slyz A., Devriendt J., Pogosyan D., 2012, MNRAS, 423, 3616

Dubois Y., Pichon C., Devriendt J., Silk J., Haehnelt M., Kimm T., Slyz A., 2013, MNRAS, 428, 2885

Fabian A. C., 2012, ARA\&A, 50, 455

Fan X., et al., 2006, AJ, 131, 1203

Farrah D., Priddey R., Wilman R., Haehnelt M., McMahon R., 2004, ApJ, 611, L13

Faucher-Giguère C.-A., Feldmann R., Quataert E., Kereš D., Hopkins P. F., Murray N., 2016, MNRAS, 461, L32

Feng Y., Di Matteo T., Croft R., Khand ai N., 2014, MNRAS, 440, 1865

Feng Y., Di-Matteo T., Croft R. A., Bird S., Battaglia N., Wilkins S., 2016, MNRAS, 455, 2778

Ferrara A., Salvadori S., Yue B., Schleicher D., 2014, MNRAS, 443, 2410

Feruglio C., et al., 2018, A\&A, 619, A39

Fiacconi D., Mayer L., Madau P., Lupi A., Dotti M., Haardt F., 2017, MNRAS, 467, 4080

Fiore F., et al., 2017, A\&A, 601, A143

Förster Schreiber N. M., et al., 2019, ApJ, 875, 21

Gallerani S., Fan X., Maiolino R., Pacucci F., 2017a, Publ. Astron. Soc. Australia, 34, e022

Gallerani S., et al., 2017b, MNRAS, 467, 3590

Gallerani S., Pallottini A., Feruglio C., Ferrara A., Maiolino R., Vallini L., Riechers D. A., Pavesi R., 2018, MNRAS, 473, 1909

Genzel R., et al., 2015, ApJ, 800, 20

Giallongo E., et al., 2015, A\&A, 578, A83 


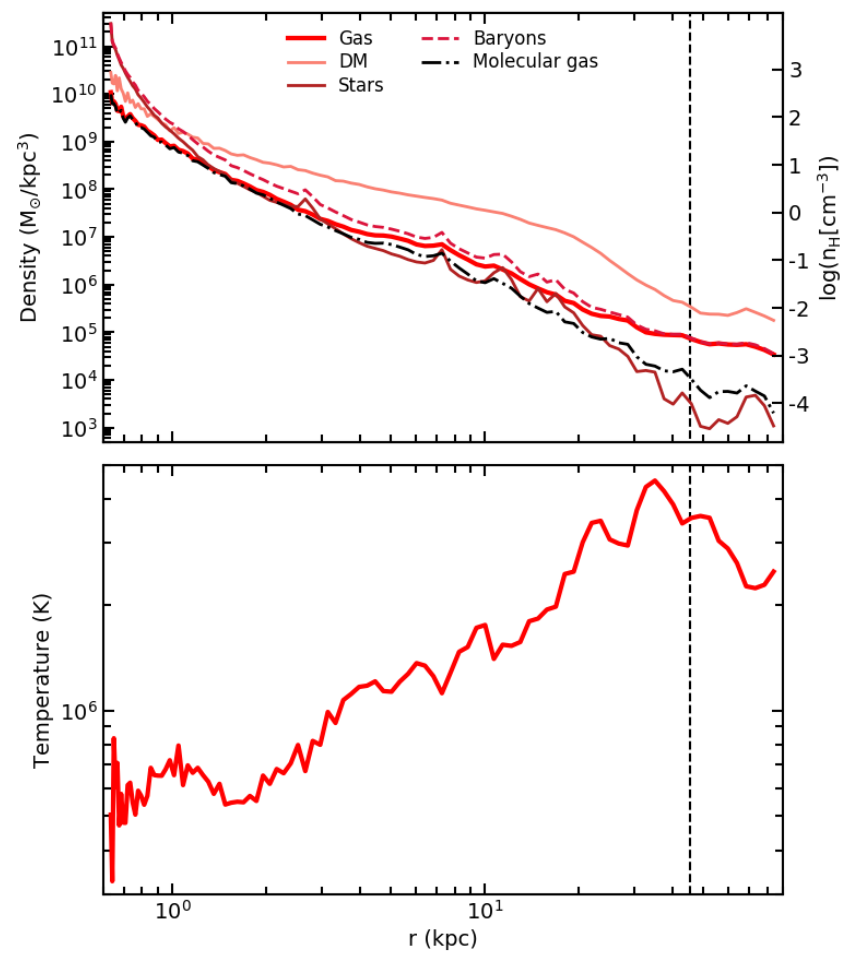

Figure B3. Density and temperature radial profiles within twice the virial radius in the simulation $S F_{-}$only, at redshift $z=6$. We show the density profile of gas (total and molecular), stars, DM, and baryons (top panel), and the mass-weighted, temperature profile (bottom panel). The vertical, dashed black line highligths the virial radius of the most massive subhalo. Same as Figure 6, but for the simulation SF_only.

Ginolfi M., et al., 2020, A\&A, 633, A90

Guo Q., White S., Li C., Boylan-Kolchin M., 2010, MNRAS, 404, 111

Haardt F., Madau P., 2001, in Neumann D. M., Tran J. T. V., eds, Clusters of Galaxies and the High Redshift Universe Observed in X-rays. CEA, Saclay, p.64. (arXiv: astro-ph/0106018)

Habouzit M., Volonteri M., Somerville R. S., Dubois Y., Peirani S., Pichon C., Devriendt J., 2019, MNRAS, 489, 1206

Hahn O., Abel T., 2011, MNRAS, 415, 2101

Hassan S., Davé R., Mitra S., Finlator K., Ciardi B., Santos M. G., 2018, MNRAS, 473, 227

Heckman T. M., Alexandroff R. M., Borthakur S., Overzier R., Leitherer C., 2015, ApJ, 809, 147

Hirschmann M., Dolag K., Saro A., Bachmann L., Borgani S., Burkert A., 2014, MNRAS, 442, 2304

Ho L. C., 2007, ApJ, 669, 821

Hoyle F., Lyttleton R. A., 1939, Proceedings of the Cambridge Philosophical Society, 35, 405

Huang K.-W., Ni Y., Feng Y., Di Matteo T., 2020, MNRAS, 496, 1

Inayoshi K., Haiman Z., Ostriker J. P., 2016, MNRAS, 459, 3738

Jiang L., et al., 2016, ApJ, 833, 222

Juarez Y., Maiolino R., Mujica R., Pedani M., Marinoni S., Nagao T., Marconi A., Oliva E., 2009, A\&A, 494, L25

Karakas A. I., 2010, MNRAS, 403, 1413

Khalatyan A., Cattaneo A., Schramm M., Gottlöber S., Steinmetz M., Wisotzki L., 2008, MNRAS, 387, 13

Khandai N., Feng Y., DeGraf C., Di Matteo T., Croft R. A. C., 2012, MNRAS, 423, 2397

Koptelova E., Hwang C.-Y., Yu P.-C., Chen W.-P., Guo J.-K., 2017, Scientific Reports, 7, 41617

Kormendy J., Ho L. C., 2013, Annual Review of Astronomy and Astrophysics, 51, 511
Lamastra A., Menci N., Maiolino R., Fiore F., Merloni A., 2010, MNRAS, 405, 29

Latif M. A., Ferrara A., 2016, Publ. Astron. Soc. Australia, 33, e051

Li J., et al., 2020, ApJ, 889, 162

Lupi A., Volonteri M., Decarli R., Bovino S., Silk J., Bergeron J., 2019, MNRAS, 488, 4004

Madau P., Haardt F., Dotti M., 2014, ApJ, 784, L38

Maio U., Borgani S., Ciardi B., Petkova M., 2018, arXiv e-prints,

Maiolino R., et al., 2005, A\&A, 440, L51

Maiolino R., et al., 2012, MNRAS, 425, L66

Marconi A., Hunt L. K., 2003, ApJ, 589, L21

Martin C. L., 2005, ApJ, 621, 227

Matsuoka Y., et al., 2016, ApJ, 828, 26

Matsuoka Y., et al., 2018, ApJ, 869, 150

Matsuoka Y., et al., 2019a, ApJ, 872, L2

Matsuoka Y., et al., 2019b, ApJ, 883, 183

Mayer L., Bonoli S., 2019, Reports on Progress in Physics, 82, 016901

Mayer L., Kazantzidis S., Escala A., Callegari S., 2010, Nature, 466, 1082

Mazzucchelli C., et al., 2017, ApJ, 849, 91

McConnell N. J., Ma C.-P., 2013, ApJ, 764, 184

McNamara B. R., Nulsen P. E. J., 2007, ARA\&A, 45, 117

Merloni A., et al., 2010, ApJ, 708, 137

Meyer R. A., Bosman S. E. I., Kakiichi K., Ellis R. S., 2019, MNRAS, 483, 19

Mortlock D. J., et al., 2011, Nature, 474, 616

Moster B. P., Somerville R. S., Maulbetsch C., van den Bosch F. C., Macciò A. V., Naab T., Oser L., 2010, ApJ, 710, 903

Murante G., Monaco P., Giovalli M., Borgani S., Diaferio A., 2010, MNRAS, 405, 1491

Murante G., Monaco P., Borgani S., Tornatore L., Dolag K., Goz D., 2015, MNRAS, 447, 178

Nanni R., Vignali C., Gilli R., Moretti A., Brand t W. N., 2017, A\&A, 603, A128

Nanni R., et al., 2018, A\&A, 614, A121

Ni Y., Di Matteo T., Feng Y., Croft R. A. C., Tenneti A., 2018, MNRAS, 481,4877

Novak M., et al., 2019, ApJ, 881, 63

Novak M., et al., 2020, ApJ, 904, 131

Novikov I. D., Thorne K. S., 1973, in Black Holes (Les Astres Occlus). pp 343-450

Olsen K., Greve T. R., Narayanan D., Thompson R., Davé R., Niebla Rios L., Stawinski S., 2017, ApJ, 846, 105

Onoue M., et al., 2017, ApJ, 847, L15

Ostriker J. P., Choi E., Ciotti L., Novak G. S., Proga D., 2010, ApJ, 722, 642

Pacucci F., Ferrara A., 2015, MNRAS, 448, 104

Page M. J., Simpson C., Mortlock D. J., Warren S. J., Hewett P. C., Venemans B. P., McMahon R. G., 2014, MNRAS, 440, L91

Pensabene A., Carniani S., Perna M., Cresci G., Decarli R., Maiolino R., Marconi A., 2020, arXiv e-prints, p. arXiv:2002.00958

Pillepich A., et al., 2018a, MNRAS, 473, 4077

Pillepich A., et al., 2018b, MNRAS, 475, 648

Planck Collaboration et al., 2016, A\&A, 594, A13

Pons E., McMahon R. G., Simcoe R. A., Banerji M., Hewett P. C., Reed S. L., 2019, MNRAS, 484, 5142

Pons E., McMahon R. G., Banerji M., Reed S. L., 2020, MNRAS, 491, 3884

Portinari L., Kotilainen J., Falomo R., Decarli R., 2012, MNRAS, 420, 732

Puchwein E., Springel V., 2013, MNRAS, 428, 2966

Ragone-Figueroa C., Granato G. L., Murante G., Borgani S., Cui W., 2013, MNRAS, 436, 1750

Rahmati A., Schaye J., Bower R. G., Crain R. A., Furlong M., Schaller M., Theuns T., 2015, MNRAS, 452, 2034

Regan J. A., Downes T. P., Volonteri M., Beckmann R., Lupi A., Trebitsch M., Dubois Y., 2019, MNRAS, 486, 3892

Romano D., Karakas A. I., Tosi M., Matteucci F., 2010, A\&A, 522, A32

Rosas-Guevara Y. M., et al., 2015, MNRAS, 454, 1038

Salmon B., et al., 2015, ApJ, 799, 183

Salvestrini F., Risaliti G., Bisogni S., Lusso E., Vignali C., 2019, A\&A, 631, A 120 
Sarria J. E., Maiolino R., La Franca F., Pozzi F., Fiore F., Marconi A., Vignali C., Comastri A., 2010, A\&A, 522, L3

Schaye J., et al., 2015, MNRAS, 446, 521

Scholtz J., et al., 2020, MNRAS, 492, 3194

Shakura N. I., Sunyaev R. A., 1973, A\&A, 24, 337

Shao Y., et al., 2019, ApJ, 876, 99

Shemmer O., et al., 2006, ApJ, 644, 86

Shields G. A., Menezes K. L., Massart C. A., Vand en Bout P., 2006, ApJ, 641,683

Sijacki D., Springel V., Di Matteo T., Hernquist L., 2007, MNRAS, 380, 877

Sijacki D., Springel V., Haehnelt M. G., 2009, MNRAS, 400, 100

Sądowski A., Gaspari M., 2017, MNRAS, 468, 1398

Smidt J., Whalen D. J., Johnson J. L., Surace M., Li H., 2018, ApJ, 865, 126

Solomon P. M., Vanden Bout P. A., 2005, ARA\&A, 43, 677

Sorini D., Davé R., Anglés-Alcázar D., 2020, arXiv e-prints, p. arXiv:2005.08971

Springel V., 2005, MNRAS, 364, 1105

Springel V., Hernquist L., 2003, MNRAS, 339, 289

Springel V., White S. D. M., Tormen G., Kauffmann G., 2001, MNRAS, 328,726

Springel V., Di Matteo T., Hernquist L., 2005, MNRAS, 361, 776

Stanley F., Jolly J. B., König S., Knudsen K. K., 2019, A\&A, 631, A78

Steinborn L. K., Dolag K., Hirschmann M., Prieto M. A., Remus R.-S., 2015, MNRAS, 448, 1504

Sugahara Y., Ouchi M., Harikane Y., Bouché N., Mitchell P. D., Blaizot J., 2019, ApJ, 886, 29

Tang J.-J., et al., 2019, MNRAS, 484, 2575

Thielemann F.-K., et al., 2003, in Hillebrandt W., Leibundgut B., eds, From Twilight to Highlight: The Physics of Supernovae. p. 331, doi:10.1007/10828549_46

Tornatore L., Borgani S., Dolag K., Matteucci F., 2007, MNRAS, 382, 1050

Trakhtenbrot B., Lira P., Netzer H., Cicone C., Maiolino R., Shemmer O., 2017a, ApJ, 836, 8

Trakhtenbrot B., Volonteri M., Natarajan P., 2017b, ApJ, 836, L1

Trebitsch M., et al., 2020a, arXiv e-prints, p. arXiv:2002.04045

Trebitsch M., Volonteri M., Dubois Y., 2020b, MNRAS, 494, 3453

Valentini M., Murante G., Borgani S., Monaco P., Bressan A., Beck A. M., 2017, MNRAS, 470, 3167

Valentini M., Bressan A., Borgani S., Murante G., Girardi L., Tornatore L., 2018, MNRAS, 480, 722

Valentini M., Borgani S., Bressan A., Murante G., Tornatore L., Monaco P., 2019, MNRAS, 485, 1384

Valentini M., et al., 2020, MNRAS, 491, 2779

Valiante R., Schneider R., Salvadori S., Gallerani S., 2014, MNRAS, 444, 2442

Veilleux S., Cecil G., Bland-Hawthorn J., 2005, ARA\&A, 43, 769

Venemans B. P., et al., 2013, ApJ, 779, 24

Venemans B. P., et al., 2015, ApJ, 801, L11

Venemans B. P., Walter F., Zschaechner L., Decarli R., De Rosa G., Findlay J. R., McMahon R. G., Sutherland W. J., 2016, ApJ, 816, 37

Venemans B. P., et al., 2017a, ApJ, 837, 146

Venemans B. P., et al., 2017b, ApJ, 845, 154

Venemans B. P., et al., 2017c, ApJ, 851, L8

Venemans B. P., et al., 2018, ApJ, 866, 159

Venemans B. P., Neeleman M., Walter F., Novak M., Decarli R., Hennawi J. F., Rix H.-W., 2019, ApJ, 874, L30

Vito F., et al., 2019, A\&A, 630, A118

Vogelsberger M., et al., 2014, MNRAS, 444, 1518

Volonteri M., 2010, A\&ARv, 18, 279

Volonteri M., Bellovary J., 2012, Reports on Progress in Physics, 75, 124901

Volonteri M., Gnedin N. Y., 2009, ApJ, 703, 2113

Volonteri M., Reines A. E., 2016, ApJ, 820, L6

Volonteri M., Silk J., Dubus G., 2015, ApJ, 804, 148

Walter F., Carilli C., Bertoldi F., Menten K., Cox P., Lo K. Y., Fan X., Strauss M. A., 2004, ApJ, 615, L17

Wang R., et al., 2008, ApJ, 687, 848

Wang R., et al., 2010, ApJ, 714, 699
Wang S. X., et al., 2013, ApJ, 778, 179

Wang R., et al., 2016, ApJ, 830, 53

Wang F., Wang R., Fan X., Wu X.-B., Yang J., Neri R., Yue M., 2019, ApJ, 880,2

Weinberger R., et al., 2017, MNRAS, 465, 3291

Wiersma R. P. C., Schaye J., Smith B. D., 2009, MNRAS, 393, 99

Willott C. J., et al., 2010, AJ, 140, 546

Willott C. J., Bergeron J., Omont A., 2017, ApJ, 850, 108

Wolfe A. M., Gawiser E., Prochaska J. X., 2005, ARA\&A, 43, 861

Woosley S. E., Weaver T. A., 1995, ApJS, 101, 181

Wurster J., Thacker R. J., 2013, MNRAS, 431, 2513

Yoon Y., Im M., Hyun M., Jun H. D., Hwang N., Kim M., Park B.-G., 2019, ApJ, 871, 57

Zhu Q., Li Y., Li Y., Maji M., Yajima H., Schneider R., Hernquist L., 2020, arXiv e-prints, p. arXiv:2012.01458

This paper has been typeset from a $\mathrm{T}_{\mathrm{E}} \mathrm{X} / \mathrm{L} \mathrm{AT} \mathrm{E} \mathrm{X}$ file prepared by the author. 